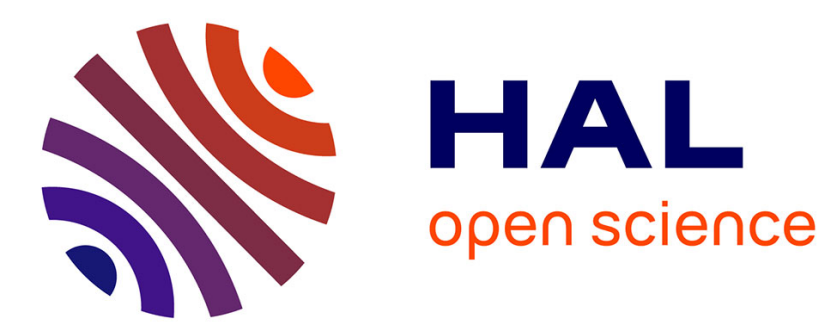

\title{
On tail dependence coefficients of transformed multivariate Archimedean copulas
}

Elena Di Bernardino, Didier Rullière

\section{To cite this version:}

Elena Di Bernardino, Didier Rullière. On tail dependence coefficients of transformed multivariate Archimedean copulas. Fuzzy Sets and Systems, 2016, 284, pp.89-112. 10.1016/j.fss.2015.08.030 . hal-00992707v2

\section{HAL Id: hal-00992707 \\ https://hal.science/hal-00992707v2}

Submitted on 20 Oct 2014

HAL is a multi-disciplinary open access archive for the deposit and dissemination of scientific research documents, whether they are published or not. The documents may come from teaching and research institutions in France or abroad, or from public or private research centers.
L'archive ouverte pluridisciplinaire HAL, est destinée au dépôt et à la diffusion de documents scientifiques de niveau recherche, publiés ou non, émanant des établissements d'enseignement et de recherche français ou étrangers, des laboratoires publics ou privés. 


\title{
On tail dependence coefficients of transformed multivariate Archimedean copulas
}

\author{
Elena Di Bernardino*1 and Didier Rullière ${ }^{\dagger 2}$ \\ ${ }^{1}$ Conservatoire National des Arts et Métiers, Département IMATH, EA4629, Paris Cedex 03, France. \\ ${ }^{2}$ Université de Lyon, Université Lyon 1, ISFA, Laboratoire SAF, EA2429, 69366 Lyon, France.
}

\begin{abstract}
This paper presents the impact of a class of transformations of copulas in their upper and lower multivariate tail dependence coefficients. In particular we focus on multivariate Archimedean copulas. In the first part of this paper, we calculate multivariate tail dependence coefficients when the generator of the considered copula exhibits some regular variation properties, and we investigate the behaviour of these coefficients in cases that are close to tail independence. This first part exploits previous works of Charpentier and Segers (2009) and extends some results of Juri and Wüthrich (2003) and De Luca and Rivieccio (2012). In the second part of the paper we analyse the impact in the upper and lower multivariate tail dependence coefficients of a large class of transformations of dependence structures. These results are based on the transformations exploited by Di Bernardino and Rullière (2013a) and Di Bernardino and Rullière (2013b). We extend some bivariate results of Durante et al. (2010) in a multivariate setting by calculating multivariate tail dependence coefficients for transformed copulas. We obtain new results under specific conditions involving regularly varying hazard rates of components of the transformation. In the third part, we show the utility of using transformed Archimedean copulas, as they permit to build Archimedean generators exhibiting any chosen couple of lower and upper tail dependence coefficients. Furthermore, using results in Larsson and Nešlehová (2011), we detail the extreme behaviour of the transformed radial part of Archimedean copulas. At last, we explain possible applications with Markov chains with specific dependence structure.
\end{abstract}

Keywords: Archimedean copulas, tail dependence coefficients, regular variation, transformations of Archimedean copulas.

2010 Mathematics Subject Classification: 60E0562E20; 62H20

\section{Introduction}

Tail problem Depending upon targeted applications, understanding the tail behaviour of a copula is of great importance. In many practical problems, like hydrology, finance, insurance, etc. one needs to understand the risk of simultaneous threshold crossing for the considered random variables. Tail dependence measurements have been proposed in the literature to explain the asymptotic probability that all random variables in a given set become large, given that random variables of another set are also large. For example, the probability that losses of some financial derivatives are important, given that losses of other derivatives are also important. The whole field of Extreme Value Theory is especially interested in such coefficients. In particular some bivariate tail dependence coefficients have been introduced by Sibuya (1960). Multivariate dependence coefficients are discussed in De Luca and Rivieccio (2012) and Li (2009). Statistical literature is also available for tail coefficients, see e.g. Joe (1997a). More detailed studies are available in the Archimedean case, where some results on the conditional joint distribution of the considered random variables are given. The interested reader is referred to Charpentier and Segers $(2009)$ for a very precise analysis of the Archimedean copulas tail behaviour, including many developments in the (difficult) cases that are close to asymptotic independence. Larsson and Nešlehová (2011) proved that the extremal behaviour of a $d$-dimensional Archimedean copulas can be deduced from the associated stochastic representation proposed in McNeil and Nešlehová (2009).

\footnotetext{
*elena.di_bernardino@cnam.fr

$\dagger$ didier.rulliere@univ-lyon1.fr
} 
Transformations of copulas Transformations of copulas are based on an initial copula $C_{0}$ and on a transformation function $T$. The idea is to exploit characteristics of both functions, $C_{0}$ and $T$, to build new classes of copulas. The main interest of transformations is that, under suitable conditions, it becomes easy to get analytical expressions for level curves of the copula, as well as some properties that are useful for estimating parameters of the transformation, as detailed for example in Di Bernardino and Rullière (2013a) or Di Bernardino and Rullière (2013b).

Different types of transformations can be found in the literature, cf. Valdez and Xiao (2011) or Michiels and De Schepper (2012) for a review of some existing transforms. See also Durante and Sempi (2005), Klement et al. (2005a) and Klement et al. (2005b), for transformations in the bivariate case. For transformations based on mixtures, see e.g. Morillas (2005).

The transformations of copulas that will be considered here are relying on transformation functions that are described in Bienvenüe and Rullière (2011). Some adaptations of such transformations to copulas are given in Di Bernardino and Rullière (2013a) or Di Bernardino and Rullière (2013b), dealing mainly with statistical estimation of these transformations. In particular, we will consider transformations that permit to transform Archimedean copulas into other Archimedean copulas. Notice that non-parametric estimation of these transformations can be linked to estimation of the generator, and several non-parametric estimators of the generator of Archimedean copulas are available in the literature (see e.g. Dimitrova et al. (2008), Genest et al. (2011), Di Bernardino and Rullière $(2013 \mathrm{~b}))$.

Despite their utility, there are however few works on tail transformations of Archimedean copulas. Such knowledge would help building new classes of Archimedean copulas exhibiting desired tail behaviour. As one will see in this paper, the flexibility of transformed Archimedean copula, coupled with a good knowledge of tail dependence coefficients, will be the starting point of the construction of copulas with given tail dependence coefficients.

Copulas with given tail dependence coefficients Estimating the dependence structure of a multivariate data is not an easy task, because non-parametric estimation may lack good representations of the tail dependence, whereas parametric representations may lack good representation of the central part of the copula. In particular, it has been shown that most non-parametric estimators have difficulties to capture tail dependence. For the special case of an Archimedean copula $C$, some estimators are based on the diagonal section $\delta_{C}(u):=C(u, \ldots, u), u \in[0,1]$. In dimension $d$, conditions satisfied by a diagonal section are given in Erdely et al. (2014) and existence of a copula with given diagonal section is recalled in their Theorem A. The uniqueness of a copula having a given diagonal section is only ensured for copulas having derivative $\delta_{C}^{\prime}(1-)=d$, this condition being referred as Frank's condition in the above mentioned article. In the dimension $d=2$, one can find counterexamples showing that for an Archimedean copula $C$ presenting upper tail dependence, the diagonal does not characterize uniquely its generator $\phi$ (see Alsina et al. (2006), Section 3.8, when $\phi^{\prime}(0)=-\infty$, or equivalently $\delta_{C}^{\prime}\left(1^{-}\right)<2$ ). It results that in the general case, estimators based on diagonal section only use partial information about the dependence and thus might not be efficient to capture tail dependence (see Hofert et al. (2011)). The estimation of dependence structure thus raises some problems for the tail. Coping with such problems requires a good knowledge of tail dependence behaviour. It also requires some specific ways to use tail dependence measures.

It thus seems that there is a need to propose a flexible parametric estimation of the generator of an Archimedean copula with given tail dependence coefficients. Such result would help adjusting parametrically both the tail and the central part of the considered copula. The first step is to be able to produce not only bivariate but multivariate tail dependence coefficients for transformed copulas. The second step is to be able to link these coefficients with parameters of a transformation, and to adapt the estimation using these coefficients. One can imagine for example starting from a copula exhibiting a good fit on the central part of the multivariate data, and applying a transformation to improve the fit of the tails. Or starting from a copula exhibiting a good fit on the tails and distorting it in order to improve its central part. Or more generally finding the best transformation $T$ to fit both the tails and the central part of a given multivariate data-set, starting from a given copula $C_{0}$. In the following, we will show that for some particular transformations and starting from some particular initial Archimedean copulas, it is possible to produce Archimedean copulas having tunable regular variation properties, and thus to get specific targeted lower and upper tail coefficients.

Organization of the paper The paper is organized as follow: In Section 1, we present multivariate tail dependence coefficients for copulas, starting from definition in De Luca and Rivieccio (2012). In Section 2, we present some suitable Regular Variation properties for Archimedean copulas and we provide the multivariate tail dependence coefficients introduced before, for these regular varying generators. Particular attention is devoted to the 
interesting asymptotic independent case. Indeed under some supplementary regular conditions, it is possible to quantify the rate of convergence toward 0 of the multivariate upper and lower tails dependence coefficients. The impact of the considered transformations on the regular generator of Archimedean copulas is considered in Section 3. In particular we give tail dependence coefficients for these transformed copulas. We obtain new results under specific conditions involving regularly varying hazard rates of components of the transformation. In Section 4 , we propose a methodology to parametrically estimate a generator given tail dependence coefficients. At last, a good knowledge on the tail behaviour of the generator of Archimedean copulas allows to get supplementary results on transformed radial part of Archimedean copulas (see Section 5.1 and results in Larsson and Nešlehová (2011)) and on transformed stationary Markov chains exhibiting specific dependence structure (Section 5.2).

\section{Multivariate tail dependence coefficients}

Assume that we have a $d$-dimensional non-negative real-valued random vector $\mathbf{X}=\left(X_{1}, \ldots, X_{d}\right)$. Denote its multivariate distribution function by $F: \mathbb{R}^{d} \rightarrow[0,1]$ with continuous univariate margins $F_{i}\left(x_{i}\right)=P\left(X_{i} \leq x_{i}\right)$, for $i=1, \ldots, d$. Sklar's Theorem (1959) is a well-known result which states that for any random vector $\mathbf{X}$, its multivariate distribution function has the representation

$$
F\left(x_{1}, \ldots, x_{d}\right)=C\left(F_{1}\left(x_{1}\right), \ldots, F_{d}\left(x_{d}\right)\right)
$$

where $C$ is called the copula. Effectively, it is a distribution function on the $d$-cube $[0,1]^{d}$ with uniform margins and it links the univariate margins to their full multivariate distribution. In the case where we have a continuous random vector, we know that $U_{i}=F_{i}\left(X_{i}\right)$ is an uniform random variable so that we can write

$$
C\left(u_{1}, \ldots, u_{d}\right)=F\left(F_{1}^{-1}\left(u_{1}\right), \ldots, F_{d}^{-1}\left(u_{d}\right)\right),
$$

to be the unique copula associated with $\mathbf{X}$, with quantile functions defined by $F_{i}^{-1}(p)=\inf \left\{x \in \mathbb{R}: F_{i}(x) \geq p\right\}$, for $p \in(0,1)$.

The tail dependence of bivariate copulas has been discussed extensively in recent statistics literature (see for instance Joe (1997a)). In particular the bivariate tail dependence coefficients were introduced by Sibuya (1960) and play a role in the bivariate Extreme Value Theory. The tail dependence coefficients in the general multivariate case can be expressed as follows (as defined in De Luca and Rivieccio (2012), Li (2009)).

Definition 1.1 (Multivariate tail dependence coefficients) Assume that the considered copula $C$ is the distribution of some random vector $\boldsymbol{U}:=\left(U_{1}, \ldots, U_{d}\right)$. Denote $I=\{1, \ldots, d\}$ and consider two non-empty subsets $I_{h} \subset I$ and $\bar{I}_{h}=I \backslash I_{h}$ of respective cardinal $h \geq 1$ and $d-h \geq 1$. Provided that the limits exist, a multivariate version of classical bivariate tail dependence coefficients is given by

$$
\left\{\begin{array}{l}
\lambda_{L}^{I_{h}, \bar{I}_{h}}=\lim _{u \rightarrow 0^{+}} \mathbb{P}\left[U_{i} \leq u, i \in I_{h} \mid U_{i} \leq u, i \in \bar{I}_{h}\right], \\
\lambda_{U}^{I_{h}, \bar{I}_{h}}=\lim _{u \rightarrow 1^{-}} \mathbb{P}\left[U_{i} \geq u, i \in I_{h} \mid U_{i} \geq u, i \in \bar{I}_{h}\right] .
\end{array}\right.
$$

Note that the existence of such coefficients is not guaranteed. As an example, if there exists a value $\epsilon \in(0,1)$ such that $\mathbb{P}\left[U_{i} \leq u, i \in \bar{I}_{h}\right]=0$ for all $u<\epsilon$, or if $\mathbb{P}\left[U_{i} \geq u, i \in \bar{I}_{h}\right]=0$ for all $u>1-\epsilon$. Lower tail dependence coefficients cannot be defined for copulas exhibiting a non-degenerated zero set (see Nelsen (1999), Section 4). If for all $I_{h} \subset I, \lambda_{L}^{I_{h}, \bar{I}_{h}}=0$, (resp. $\lambda_{U}^{I_{h}, \bar{I}_{h}}=0$ ) then we say $\boldsymbol{U}$ is lower tail independent (resp. upper tail independent).

From Definition 1.1, the multivariate tail dependence coefficients describe the relative deviation of upper- (or lower-) tail probabilities of a random vector from similar orthant tail probabilities of a subset of its components. They can be used in the study of dependence among extreme values (see, for instance, Li (2009)). In particular, for the independence copula we have $\lambda_{L}=\lambda_{U}=0$ (tail independence) and for the comonotonic copula we have $\lambda_{L}=\lambda_{U}=1$ (perfect tail dependence).

One can easily check that Definition 1.1 corresponds to the classical definition in the bivariate case where $d=2$, with necessarily $h=d-h=1$. In this case, due to uniform margins of $\mathbf{U}$, the bivariate lower and upper tail coefficients $\lambda_{U}$ and $\lambda_{L}$ do not depend on the choice of subsets $I_{h}$ and $\bar{I}_{h}$. They can also be written using the diagonal section $\delta_{C}(u)=C(u, u)$ of the associated copula $C$ and its derivative $\delta_{C}^{\prime}$ (see e.g. Nelsen (1999), Nelsen 
et al. (2008)):

$$
\left\{\begin{array}{l}
\lambda_{L}=\lim _{u \rightarrow 0^{+}} \mathbb{P}\left[U_{2} \leq u \mid U_{1} \leq u\right]=\delta_{C}^{\prime}\left(0^{+}\right), \\
\lambda_{U}=\lim _{u \rightarrow 1^{-}} \mathbb{P}\left[U_{2}>u \mid U_{1}>u\right]=2-\delta_{C}^{\prime}\left(1^{-}\right) .
\end{array}\right.
$$

Note that in the bivariate case there exists other expressions where the bivariate lower and upper tail coefficients can be defined using limits of the logarithm of the diagonal section of the copula (see, for example, Coles et al. $(1999)$ ).

Note that these tail dependence coefficients are a copula-based dependence measures. Unfortunately, they have some drawback. For example, they evaluate the copula $C$ solely on its diagonal section. In other words, the limiting behaviour may be very different if we tend to the copula's lower left (resp. copula's upper right) corner on a different route than on the main diagonal. Regarding this drawback and other pitfalls, the reader may consult Schlather (2001), Abdous et al. (2005) and Frahm et al. (2005).

Coefficients in Definition 1.1 can be easily written when $C$ is an exchangeable copula, and in particular for a $d$-Archimedean copula with generator $\phi$, i.e.,

$$
C\left(u_{1}, \ldots, u_{d}\right)=\phi\left(\phi^{-1}\left(u_{1}\right)+\ldots+\phi^{-1}\left(u_{d}\right)\right), \text { for } u_{1}, \ldots, u_{d} \in(0,1] .
$$

The generator $\phi(t)$ is a continuous, decreasing and convex function of $t$, with $\phi(0)=1$. From Theorem 2.2 in McNeil and Nešlehová (2009), $C$ in Equation (2) is a $d$-dimensional copula if and only if its generator $\phi$ is $d$-monotone on $[0, \infty)$ (for details the reader is referred to McNeil and Nešlehová (2009)). Required proprieties on generator $\phi$ an its inverse $\psi$ are gathered in Assumption 1.1 below.

Assumption 1.1 (Considered generators) Consider a valid Archimedean generator $\phi$, thus $d-$ monotone on $[0, \infty)$ with $\phi(0)=1$. One assumes that $\phi$ is differentiable and that $\phi$ is a strict generator, i.e. strictly positive with limit $\lim _{t \rightarrow+\infty} \phi(t)=0$ (see Section 4 in Nelsen, 1999).

Under Assumption 1.1, the generator $\phi$ has a proper inverse that will be denoted

$$
\psi(t)=\phi^{-1}(t), \quad t \in(0,1] .
$$

Remark that $\psi$ is convex and, if $\psi$ is differentiable, then $\psi^{\prime}$ is increasing and in particular ultimately monotone. Then, for generators exhibiting some Regular Variation properties (see Section 2), Assumption 1.1 guarantees the application of the Monotone Density Theorem (see, e.g., Theorem 1.7.2 in Bingham et al. (1989) and Theorem 1.20 in Soulier (2009)).

Lemma 1.1 below naturally gives the form of multivariate lower and upper tail coefficients in the particular Archimedean copulas case (see Definition 2 in De Luca and Rivieccio (2012)). We consider here strict generators as in Assumption 1.1 to avoid some situations where the lower tail dependence coefficient does not exist.

Lemma 1.1 (Multivariate tail dependence coefficients for Archimedean copulas) For Archimedean copulas in Equation (2) with generator as in Assumption 1.1, the multivariate lower and upper tail dependence coefficients in Definition 1.1 are respectively:

$$
\left\{\begin{array}{l}
\lambda_{L}^{(h, d-h)}=\lim _{u \rightarrow 0^{+}} \frac{\psi^{-1}(d \psi(u))}{\psi^{-1}((d-h) \psi(u))}, \\
\lambda_{U}^{(h, d-h)}=\lim _{u \rightarrow 1^{-}} \frac{\sum_{i=0}^{d}(-1)^{i} C_{d}^{i} \psi^{-1}(i \psi(u))}{\sum_{i=0}^{d-h}(-1)^{i} C_{d-h}^{i} \psi^{-1}(i \psi(u))} .
\end{array}\right.
$$

\section{Tails of Archimedean copulas}

In this section we summarize different results (see Lemma $\mathrm{A}$ and Lemma $\mathrm{B}$ ) obtained in the recent literature about the tail behavior of Archimedean copulas (see, e.g., Hua and Joe (2011), Charpentier and Segers (2009), Larsson and Nešlehová (2011)). Furthermore we present a multivariate extension of Theorem 4.4 in Juri and Wüthrich (2003) and Theorem 3.9 in Juri and Wüthrich (2002) about the tail dependence coefficients for regular Archimedean copulas (see Theorem 2.1). These auxiliary results will be necessary to understand our main contribution on the multivariate tail coefficients of transformed Archimedean copulas (see Sections 3 and 44).

For the sake of simplicity, we define as regular Archimedean copulas the Archimedean copulas having a lower and upper regularly varying inverse generator $\psi$ at zero and one respectively (see Equation (3)). We define in following paragraphs regularly varying function and considered generators of regular Archimedean copulas. 


\subsection{Regular Variation}

We recall here some basic definitions of regular variation for a measurable function $f:(0, \infty) \rightarrow(0, \infty)$. We say that $f$ is regularly varying at $+\infty$ with index $\alpha$, and we denote $f \in \mathcal{R} \mathcal{V}_{\alpha}(\infty)$ if

$$
f \in \mathcal{R} \mathcal{V}_{\alpha}(\infty) \Leftrightarrow \forall s>0, \lim _{x \rightarrow+\infty} \frac{f(s x)}{f(x)}=s^{\alpha} .
$$

A survey of regular variations can be found for example in Embrechts et al. (1997), de Haan and Ferreira (2006), Soulier (2009). Main results about regular variations are presented by Bingham et al. (1989).

For $0<x<\infty$, define $x^{\infty}$ by $\infty, 1$, or 0 , according to whether $x$ is larger than, equal to, or smaller than 1 , respectively; similarly, define $x^{-\infty}$ by 0,1 , or $\infty$ according to whether $x$ is larger than, equal to, or smaller than 1 , respectively. This proper interpretation of the considered power functions, $x^{\infty}$ and $x^{-\infty}$, will be used in the following.

Regular variations can also be defined at zero or at one by requiring that simple transformations of the function are regularly varying at infinity. Let us define, for example,

$$
M:[0,1] \rightarrow[0,1], M(x)=1-x, \quad I:(0, \infty) \rightarrow(0, \infty), I(x)=\frac{1}{x}, \quad N: \mathbb{R} \rightarrow \mathbb{R}, N(x)=-x .
$$

One easily check that $M \circ M, I \circ I$ and $N \circ N$ are the identity function on considered intervals. For a function $f$ defined on $(0,1)$, we will say that $f$ is regularly varying at zero with index $\alpha$ if

$$
f \in \mathcal{R V}_{\alpha}(0) \Leftrightarrow f \circ M \in \mathcal{R} \mathcal{V}_{\alpha}(1) \Leftrightarrow f \circ I \in \mathcal{R} \mathcal{V}_{-\alpha}(\infty) \Leftrightarrow \forall s>0, \lim _{x \rightarrow 0^{+}} \frac{f(s x)}{f(x)}=s^{\alpha}
$$

We also define functions that are regularly varying at one with index $\alpha$ as

$$
f \in \mathcal{R} \mathcal{V}_{\alpha}(1) \Leftrightarrow f \circ M \circ I \in \mathcal{R} \mathcal{V}_{-\alpha}(\infty) \Leftrightarrow \forall s>0, \lim _{x \rightarrow 0^{+}} \frac{f(1-s x)}{f(1-x)}=s^{\alpha}
$$

Finally, one can say that $f$ is regularly varying with index $\alpha$ at $-\infty$ if

$$
f \in \mathcal{R} \mathcal{V}_{\alpha}(-\infty) \Leftrightarrow f \circ N \in \mathcal{R} \mathcal{V}_{\alpha}(\infty) \Leftrightarrow \forall s>0, \lim _{x \rightarrow-\infty} \frac{f(s x)}{f(x)}=s^{\alpha}
$$

\subsection{Upper and lower regular generators}

As $\psi \circ M \circ I$ is a decreasing function, if $\psi \circ M \circ I$ is regularly varying, it can only be varying with some negative index $-\alpha, \alpha \in[0,+\infty]$, so that $\psi \in \mathcal{R} \mathcal{V}_{\alpha}(1)$ requires that $\alpha \in[0,+\infty]$. We recall in Lemma A that convexity assumptions restrict the range of possible regular variation indexes for Archimedean generators.

Table 4.1 in Nelsen (1999) contains several examples of strict archimedean copulas whose inverse generators $\psi$ in (3) are regularly varying at one and zero.

Lemma A (Valid regularly varying generator) Assume that the generator $\phi$ and its inverse $\psi$ satisfy Assumption 1.1. It holds that:

i) If an inverse generator $\psi$ is regularly varying at zero with index $-r$, i.e. $\psi \in \mathcal{R} \mathcal{V}_{-r}(0)$, then necessarily $r \in[0,+\infty]$.

ii) If an inverse generator $\psi$ is regularly varying at one with index $\rho$, i.e. $\psi \in \mathcal{R} \mathcal{V}_{\rho}(1)$, then necessarily $\rho \in$ $[1,+\infty]$.

Lemma A comes down from Lemma 2.7 in Juri and Wüthrich (2002) and by applying the Hôpital's rule. Remark that the independent generator is $\psi^{\text {Indep }} \in \mathcal{R V}_{0}(0)$, with $r=0$. Furthermore, for any $r \in(0,+\infty)$, there exists a valid inverse generator $\psi$ with index $-r$ at zero. Indeed the strict Clayton generator is an example of generator valid in any dimension allowing to reach any regular variation index $-r$, with $r \in(0,+\infty)$ at zero (see Equation (21)). Furthermore for any $\rho \in[1,+\infty)$, there exists a valid inverse generator $\psi$ with index $\rho$ at one. Indeed the Gumbel generator is an example of generator valid in any dimension allowing to reach any regular variation index $\rho \in[1,+\infty)$ at one (see Equation 20). Charpentier and Segers (2009) listed different generators with $\rho=+\infty$ or $r=+\infty$ 
(see Table 1 in Charpentier and Segers (2009)). Then the bounds of the intervals for $\rho$ and $r$ in Lemma A can be reached.

For the sake of simplicity, we denote by regular generator any regularly varying Archimedean generator. We introduce here the definition of a lower and upper regular generator.

Definition 2.1 (Lower and upper regular generators) Assume that the generator $\phi$ and its inverse $\psi$ satisfy Assumption 1.1. Then

i) if $\psi$ is regularly varying at zero with index $-r$, with $r \in[0,+\infty]$, then we say that $\psi$ is a lower regular generator.

ii) if $\psi$ is regularly varying at one with index $\rho$, with $\rho \in[1,+\infty]$, then we say that $\psi$ is an upper regular generator.

A generator $\psi$ which is both an upper and lower regular generator is simply called a (full) regular generator.

Identifying regular generator is easier if we use properties in Lemma B that will be useful to calculate tail dependence coefficients.

Lemma B Let $\psi$ be the generator of an Archimedean copula and let $r \in[0,+\infty]$, then

$$
\begin{aligned}
\text { i) } \psi \in \mathcal{R} \mathcal{V}_{-r}(0) \Leftrightarrow \psi \circ I \in \mathcal{R} \mathcal{V}_{r}(\infty) \Leftrightarrow \lim _{y \rightarrow 0^{+}} \frac{y \psi^{\prime}(y)}{\psi(y)}=-r, \\
\text { ii) } \psi \in \mathcal{R} \mathcal{V}_{-r}(0) \Leftrightarrow \phi \in \mathcal{R} \mathcal{V}_{-1 / r}(\infty), \\
\text { iii) } \psi \in \mathcal{R} \mathcal{V}_{-r}(0) \Rightarrow \lim _{u \rightarrow 0^{+}} \frac{\delta^{(i)}(u)}{u}=\lim _{u \rightarrow 0^{+}}\left[\delta^{(i)}\right]^{\prime}(u)=i^{-1 / r},
\end{aligned}
$$

with proper interpretations for $r$ equal to zero or infinity, where $\delta^{(i)}(u)=\psi^{-1}(i \cdot \psi(u))$ is the diagonal section of the copula in dimension $i, u \in(0,1)$, and $\left[\delta^{(i)}\right]^{\prime}$ its derivatives. Now let $\rho \in[1,+\infty]$, then

$$
\begin{aligned}
& \text { i) } \psi \in \mathcal{R V}_{\rho}(1) \Leftrightarrow \psi \circ M \circ I \in \mathcal{R} \mathcal{V}_{-\rho}(\infty) \Leftrightarrow \lim _{z \rightarrow 1^{-}} \frac{(1-z) \psi^{\prime}(z)}{\psi(z)}=-\rho, \\
& \text { ii) } \psi \in \mathcal{R} \mathcal{V}_{\rho}(1) \Leftrightarrow M \circ \phi \in \mathcal{R} \mathcal{V}_{1 / \rho}(0) \\
& \text { iii) } \psi \in \mathcal{R} \mathcal{V}_{\rho}(1) \Rightarrow \lim _{u \rightarrow 1^{-}} \frac{1-\delta^{(i)}(u)}{1-u}=\lim _{u \rightarrow 1^{-}}\left[\delta^{(i)}\right]^{\prime}(u)=i^{1 / \rho},
\end{aligned}
$$

with proper interpretation for $\rho$ equal to infinity.

Results in Lemma B follow from the Monotone Density theorem (see Theorem 1.7.2 in Bingham et al. (1989)). The interest reader is also referred to Theorem 1 in Charpentier and Segers (2007). Note that when the diagonal section $\delta$ tends to 0 or to 1 , it is useful to get some results on the convergence speed toward 0 or 1 . From Charpentier and Segers (2009), the following Lemma 2.1 will be helpful in the case where $r=0$ or $\rho=1$.

Lemma 2.1 (Regular Variation of copula diagonal sections) Recall that the diagonal of an Archimedean copula in dimension $i \in \mathbb{N} \backslash\{0\}$ is $\delta^{(i)}(u)=\phi\left(i \cdot \phi^{-1}(u)\right)$, for $u \in(0,1)$. Assume that the generator of the copula is as in Definition 2.1, and notice that the generator $\phi$ of the copula can be seen as the survival function of a positive random variable. On the lower side, if the hazard rate $\mu_{\phi}=-\phi^{\prime} / \phi \in \mathcal{R} \mathcal{V}_{k-1}(\infty)$, then for $i \in \mathbb{N} \backslash\{0\}$,

$$
\delta^{(i)} \in \mathcal{R} \mathcal{V}_{z_{i}}(0) \text {, with } z_{i}=i^{k} \text { and } k \in[0,+\infty] .
$$

On the upper side, if the rate $m_{\phi}=-\phi^{\prime} /(1-\phi) \in \mathcal{R} \mathcal{V}_{-\kappa-1}(0)$, then for $i \in \mathbb{N} \backslash\{0\}$,

$$
M \circ \delta^{(i)} \in \mathcal{R} \mathcal{V}_{\zeta_{i}}(1), \text { with } \zeta_{i}=i^{-\kappa} \text { and } \kappa \in[0,+\infty] .
$$

The proof is postponed to Appendix B. 
Remark 1 In Charpentier and Segers (2009) (Theorem 3.3) the authors required that the function $-\frac{1}{D(\ln \phi)} \in \mathcal{R} \mathcal{V}_{k^{*}}$ with $k^{*} \leq 1$, where $D$ is the derivative operator. We can write, using the notation of Lemma 2.1.

$$
-\frac{1}{D(\ln \phi)}=-\frac{\phi}{\phi^{\prime}}=+\frac{1}{\mu_{\phi}} .
$$

Then the assumption in Charpentier and Segers (2009) is equivalent to $+\frac{1}{\mu_{\phi}} \in \mathcal{R} \mathcal{V}_{k^{*}}$. Finally $\mu_{\phi} \in \mathcal{R} \mathcal{V}_{\bar{k}}$, with $\bar{k}=-k^{*}$ and $\bar{k} \geq-1$. So it is exactly the assumption in Lemma 2.1, i.e., $\mu_{\phi} \in \mathcal{R} \mathcal{V}_{k-1}$, with $k-1 \geq-1$ i.e. $k \geq 0$.

Remark 2 Result in Equation (4) in Lemma 2.1 (for the lower tails) will be useful in Theorem 2.2 to describe the behaviour of the lower tail coefficient in the case of lower asymptotic independence $(r=0)$. Conversely result in Equation (5) in Lemma 2.1 (for the upper tails) will not be sufficient to characterize the regular variation of upper tail coefficient in Theorem 2.3 in the case of upper asymptotic independence $(\rho=1)$. Indeed the linear combination of $\mathcal{R} \mathcal{V}_{1}(1)$ functions can be a $\mathcal{R} \mathcal{V}_{j}(1)$ function with $j>1$, due to eventual compensations between the terms of the sum. This is consistent with the fact that in a upper asymptotic independent case the diagonal section of a copula does not characterize uniquely its generator (see for instance Embrechts and Hofert (2011), Alsina et al. (2006)). For this reason, we need to introduce some different assumptions in Theorem 2.3 below to obtain the desired result.

Furthermore we can prove the following result for the range of index $k$ and $\kappa$ in Lemma 2.1

Lemma 2.2 (Hazard rates) Assume that $\psi \in \mathcal{R} \mathcal{V}_{-r}(0), r \in[0,+\infty]$, and that $\mu_{\phi}=-\phi^{\prime} / \phi \in \mathcal{R} \mathcal{V}_{k-1}(\infty)$, $k \in[0,+\infty]$, then

$$
r \in(0,+\infty) \Rightarrow k=0 .
$$

Assume that $\psi \in \mathcal{R} \mathcal{V}_{\rho}(1), \rho \in[1,+\infty]$, and that $m_{\phi}=-\phi^{\prime} /(1-\phi) \in \mathcal{R} \mathcal{V}_{-\kappa-1}(0), \kappa \in[0,+\infty]$, then

$$
\rho \in[1,+\infty) \Rightarrow \kappa=0 .
$$

Result before comes down from Lemma B. Remark for instance that in the Gumbel case $r=0$ and $k \in(0,1]$ (see Appendix A. When $\rho=+\infty$, the copula has upper tail comonotonicity (see Proposition 3 of Hua and Joe (2012)), and a nontrivial example is the two-parameter family BB3 copula of Joe (1997b) with support in the whole unit cube. Charpentier and Segers (2009) provide an example (with a partial support) of generator such that $\rho=+\infty$ (see generator (18) in Table 1 in Charpentier and Segers (2009)). In both these two cases we find $\kappa=0$.

\subsection{Tail dependence coefficients for regular Archimedean copulas}

We now focus on the multivariate upper and lower tail coefficients for regular Archimedean copulas.

Theorem 2.1 (Upper and lower tail coefficients) Let $\psi$ be an inverse generator as in Definition 2.1 i), with in particular $\psi \in \mathcal{R V}_{-r}(0)$, with $r \in[0,+\infty]$. Then

$$
\lambda_{L}^{(h, d-h)}= \begin{cases}0, & \text { if } r=0, \\ d^{-1 / r}(d-h)^{1 / r}, & \text { if } r \in(0,+\infty), \\ 1, & \text { if } r=+\infty\end{cases}
$$

Let $\psi$ be an inverse generator as in Definition 2.1 ii), with in particular $\psi \in \mathcal{R} \mathcal{V}_{\rho}(1)$, with $\rho \in[1,+\infty]$, then

$$
\lambda_{U}^{(h, d-h)}= \begin{cases}0, & \text { if } \rho=1, \\ \frac{\sum_{i=1}^{d}(-1)^{i} C_{d}^{i} \cdot i^{1 / \rho}}{\sum_{i=1}^{d-h}(-1)^{i} C_{d-h}^{i} \cdot i^{1 / \rho}}, & \text { if } \rho \in(1,+\infty), \\ 1, & \text { if } \rho=+\infty .\end{cases}
$$

The proof is postponed to Appendix $\mathrm{B}$. Note that in the case where $r \in(0,+\infty)$ or $\rho \in(1,+\infty)$ this extends results that are obtained by De Luca and Rivieccio (2012) for some particular multivariate bi-parametric (MB) copulas (namely MB1 and MB7 copulas, when $r>0$ and $\rho>1$ ). In the particular bivariate case, when $d=2$ and $h=1$, Theorem 2.1 is exactly Theorem 4.4 in Juri and Wüthrich (2003), i.e.,

$$
\widetilde{\lambda}_{L}^{(1,1)}=2^{-1 / r} \quad \text { and } \quad \widetilde{\lambda}_{U}^{(1,1)}=2-2^{1 / \rho}, \quad \text { with } r \in[0,+\infty] \text { and } \rho \in[1,+\infty],
$$


with proper interpretations for $r$ equal to zero or $r, \rho$ equal to infinity.

In this sense Theorem 2.1 can be seen as a multivariate extension of Theorem 4.4 in Juri and Wüthrich $(2003)$ and Theorem 3.9 in Juri and Wüthrich (2002). From Theorem 2.1, there are essentially two categories: if $r \in(0,+\infty]$ (resp. $\rho \in(1,+\infty])$, then the lower (resp. upper) tail exhibits asymptotic dependence, while if $r=0$ (resp. $\rho=1)$, then there is asymptotic independence. However in these asymptotic independent case, under some regular conditions, it is possible to quantify the rate of convergence toward 0 of $\lambda_{L}^{(h, d-h)}(u)$ and $\lambda_{U}^{(h, d-h)}(u)$ (see Theorems 2.2 and 2.3 below).

Theorem 2.2 (case $r=0$ ) Assume that $\psi \in \mathcal{R} \mathcal{V}_{-r}(0)$, with $r=0$. Denote by $\lambda_{L}^{(h, d-h)}(u)=\frac{\delta^{(d)}(u)}{\delta^{(d-h)}(u)}$. As $\phi$ can be seen as a survival function, define the hazard rate $\mu_{\phi}=-\frac{\phi^{\prime}}{\phi}$. If one assumes that $\mu_{\phi} \in \mathcal{R} \mathcal{V}_{k-1}(\infty)$, for $k \in[0,+\infty)$, then

$$
\lambda_{L}^{(h, d-h)}(u)=\frac{\delta^{(d)}(u)}{\delta^{(d-h)}(u)} \in \mathcal{R} \mathcal{V}_{z}(0), \text { with } z=d^{k}-(d-h)^{k} .
$$

The proof is postponed to Appendix B. Under assumptions of Theorem 4.3 and Corollary 4.7 in Charpentier and Segers (2009) we can obtain the following result in the case $\rho=1$.

Theorem 2.3 (case $\rho=1$ ) Assume that $\psi \in \mathcal{R} \mathcal{V}_{\rho}(1)$, with $\rho=1$, and that the associated $\phi$ is a $d$ times continuously differentiable generator.

- If $(-D)^{d} \phi(0)$ is finite and not zero, where $D$ is the derivative operator, then we get

$$
\lambda_{U}^{(h, d-h)}(u) \in \mathcal{R} \mathcal{V}_{h}(1)
$$

- If $\psi^{\prime}(1)=0$ and the function $L(s):=s \frac{\mathrm{d}}{\mathrm{d} s}\left\{\frac{\psi(1-s)}{s}\right\}$ is positive and $L \in \mathcal{R} \mathcal{V}_{0}(0)$, then we get

$$
\lambda_{U}^{(h, d-h)}(u) \in \mathcal{R} \mathcal{V}_{0}(1)
$$

The proof is postponed to Appendix B. Remark that $(-D)^{d} \phi(0)<+\infty$ implies $\psi^{\prime}(1)<0$ (see Theorem 4.3 in Charpentier and Segers (2009)). In the first case of Theorem 2.3, there is upper asymptotic independence in a rather strong sense, a case which is called near independence in Ledford and Tawn (1997) (Section 4.2). Indeed in this case the upper tail coefficient goes to zero as a regular variation function of index $h \geq 1$. In the second case we are on the boundary between asymptotic independence and asymptotic dependence. Charpentier and Segers (2009) called this second case near asymptotic dependence and they provided an example of generator such that $\rho=1$ and $\psi^{\prime}(1)=0$ (see generator (23) in Table 1 in Charpentier and Segers (2009)). From Theorem 2.3, in the near asymptotic dependence case the upper tail coefficient goes to zero as a slowly variation function. In Appendix A we illustrate properties gathered in Section 2 for some usual Archimedean copulas.

\section{Tails of Transformed copulas}

\subsection{Transformations on $[0,1]$}

We take transformations $T:[0,1] \rightarrow[0,1]$, where $T$ is an increasing continuous bijection, with $T(0)=0$ and $T(1)=1$. We consider here a transformed copula $\widetilde{C}_{T, C_{0}}$, which is transformed from an initial copula $C_{0}$ using the transformation $T$, i.e.,

$$
\widetilde{C}_{T, C_{0}}\left(u_{1}, \ldots, u_{d}\right)=T \circ C_{0}\left(T^{-1}\left(u_{1}\right), \ldots, T^{-1}\left(u_{d}\right)\right) .
$$

The presence of the transformation $T$ both inside and outside the copula $C_{0}$ in Equation $(6)$ is necessary to ensure that the copula preserves uniform margins, and in particular that $\widetilde{C}_{T, C_{0}}(u, 1, \ldots, 1)=u$ as required.

Transformations of copulas are used in the recent literature as a simple way to generate new copulas from initial ones. Many types of transformations of copulas have been considered, see for example Valdez and Xiao (2011) or Michiels and De Schepper (2012) for a review of some existing transforms. Transformations of bivariate copula, semicopulas and quasi-copulas are studied in Durante and Sempi (2005). Klement et al. (2005a) and Klement et al. (2005b) focused on transformations of bivariate Archimax copulas. Transformations as in Equation (6) have been considered for example in Durrleman et al. (2000), Valdez and Xiao (2011) (Definitions 3.6, in dimension 
$d=2$ ), Hofert (2011). If we focus on the two-dimensional setting, the transformation considered in this paper corresponds to the Right Composition (RC, see Lemma 5 in Michiels and De Schepper (2012)), initially defined in Genest et al. (1998).

As remarked in Di Bernardino and Rullière (2013b), if we restrict ourselves to the case where $C_{0}$ in Equation (6) is an Archimedean copula (see Equation (2) ) then, under supplementary conditions for the transformation $T$ (see Definition 3.1 below) the transformed copula $\widetilde{C}_{T, C_{0}}$ will be Archimedean. This implies that the obtained transformed copula is still symmetric, for example. Remark that, in general, $\widetilde{C}_{T, C_{0}}$ is not necessarily a copula. Determination of sufficient and necessary conditions in order to obtain admissible transformations $T$ is fundamental to propose tractable transformations in operational problems (see for instance Di Bernardino and Rullière (2013b)). In Definition 3.1 we introduce an admissible transformation $T$ to guarantee that $\tilde{C}_{T, C_{0}}$ is also an Archimedean copula.

Definition 3.1 (Admissible transformations) Consider an initial Archimedean copula $C_{0}$ as in Equation (2), with generator $\phi_{0}$ satisfying Assumption 1.1. Let $T:[0,1] \rightarrow[0,1]$, be a continuous and increasing function with $T(0)=0$ and $T(1)=1$. We say that $T$ is an admissible transformation for the initial copula $C_{0}$ if $\widetilde{\phi}=T \circ \phi_{0}$ also satisfying Assumption 1.1 .

Then $T$ as in Definition 3.1 is such that $\widetilde{C}_{T, C_{0}}$ is a proper $d$-dimensional Archimedean copula and $\widetilde{\phi}$ is an admissible generator with differentiable inverse $\widetilde{\psi}$. Clearly Definition 3.1 gives an implicit condition, requiring the $d$-monotony of $\widetilde{\phi}=T \circ \phi_{0}$ (see McNeil and Nešlehová (2009)). For a $d$-times differentiable transformation $T$, it is possible to use Faa Di Bruno's formula to get more explicit expressions of the $d$-monotony of $\widetilde{\phi}=T \circ \phi_{0}$. These expressions are quite complex, so that we omit them for the sake of clarity. When the initial copula $C_{0}$ is the independent one, tractable necessary and sufficient admissibility conditions are given in Di Bernardino and Rullière (2013b).

Theorem 3.1 (Regularly varying transformed generator) Let $\phi_{0}$ be an initial generator as in Assumption 1.1 and $T$ be an admissible transformation as in Definition 3.1. Assume that $\psi_{0}$ is as in Definition 2.1 i) i.e., $\psi_{0} \in \mathcal{R} \mathcal{V}_{-r_{0}}(0)$, with $r_{0} \in[0,+\infty]$ and that $T \in \mathcal{R} \mathcal{V}_{\widetilde{a}}(0)$, with $\widetilde{a} \in(0,+\infty)$. Then the inverse transformed generator $\widetilde{\psi}$ is such that

$$
\widetilde{\psi} \in \mathcal{R} \mathcal{V}_{-\widetilde{r}}(0) \text { with } \widetilde{r}=\frac{r_{0}}{\widetilde{a}}
$$

Assume that $\psi_{0}$ is as in Definition 2.1 (ii) i.e., $\psi_{0} \in \mathcal{R V}_{\rho_{0}}(1)$, with $\rho_{0} \in[1,+\infty]$ and that $M \circ T \in \mathcal{R} \mathcal{V}_{\widetilde{\alpha}}(1)$ with $\widetilde{\alpha} \in\left(0, \rho_{0}\right]$. Then inverse transformed generator $\widetilde{\psi}$ is such that

$$
\widetilde{\psi} \in \mathcal{R} \mathcal{V}_{\widetilde{\rho}}(1) \text { with } \widetilde{\rho}=\frac{\rho_{0}}{\widetilde{\alpha}},
$$

and $\tilde{\rho} \in[1,+\infty]$.

The proof is postponed to Appendix B.

Theorem 3.2 (Multivariate tail coefficients of transformed Archimedean copula) Let $\phi_{0}$ be an initial generator as in Assumption 1.1 and $T$ be an admissible transformation as in Definition 3.1. Assume that $\psi_{0}$ is as in Definition 2.1 i) i.e., $\psi_{0} \in \mathcal{R} \mathcal{V}_{-r_{0}}(0)$, with $r_{0} \in[0,+\infty]$ and that $T \in \mathcal{R} \mathcal{V}_{\widetilde{a}}(0)$, with $\widetilde{a} \in(0,+\infty)$. Let $\widetilde{r}=r_{0} / \widetilde{a}$. Then the transformed multivariate lower tail dependence coefficient associated to $\widetilde{\phi}$ is given by:

$$
\widetilde{\lambda}_{L}^{(h, d-h)}= \begin{cases}\text { see Theorem } 3.4 & \text { if } \widetilde{r}=0, \\ d^{-\widetilde{a} r_{0}^{-1}(d-h)^{\widetilde{a}} r_{0}^{-1}}, & \text { if } \widetilde{r} \in(0,+\infty), \\ 1, & \text { if } \widetilde{r}=+\infty .\end{cases}
$$

Assume now that $\psi_{0}$ is as in Definition 2.1 (ii) i.e., $\psi_{0} \in \mathcal{R} \mathcal{V}_{\rho_{0}}(1)$, with $\rho_{0} \in[1,+\infty]$ and that that $M \circ T \in \mathcal{R} \mathcal{V}_{\widetilde{\alpha}}(1)$, with $\widetilde{\alpha} \in\left(0, \rho_{0}\right]$. Let $\widetilde{\rho}=\rho_{0} / \widetilde{\alpha}$, the transformed multivariate upper tail dependence coefficient associated to $\widetilde{\phi}$ is given by:

$$
\widetilde{\lambda}_{U}^{(h, d-h)}= \begin{cases}\text { see Theorem 3.5. } & \text { if } \widetilde{\rho}=1, \\ \frac{\sum_{i=1}^{d} C_{d}^{i}(-1)^{i} \cdot i^{\tilde{\alpha}} \rho_{0}^{-1}}{\sum_{i=1}^{d-h} C_{d-h}^{i}(-1)^{i} \cdot i^{\tilde{\alpha} \rho} \rho_{0}^{-1}}, & \text { if } \widetilde{\rho} \in(1,+\infty), \\ 1, & \text { if } \widetilde{\rho}=+\infty .\end{cases}
$$


These results come down from a direct application of Theorem 2.1 and Theorem 3.1 for multivariate tail coefficients with a generator with regular variation at one or at zero.

The following Theorem 3.3 will be helpful in the transformed case with $\widetilde{r}=0$, (see Theorem 3.4) or with $\widetilde{\rho}=1$ (see Theorem 3.5).

Theorem 3.3 (Transformed hazard rates) Assume that $\mu_{\phi_{0}}=-\phi_{0}^{\prime} / \phi_{0} \in \mathcal{R} \mathcal{V}_{k_{0}-1}(\infty)$, with $k_{0} \in[0,+\infty]$, then if furthermore $T \in \mathcal{R} \mathcal{V}_{\widetilde{a}}(0)$ for some index $\widetilde{a} \in(0,+\infty)$, then

$$
\mu_{\widetilde{\phi}}=-\widetilde{\phi}^{\prime} / \widetilde{\phi} \in \mathcal{R} \mathcal{V}_{\widetilde{k}-1}(\infty) \text { with } \widetilde{k}=k_{0} \text {. }
$$

Assume that $m_{\phi_{0}}=-\phi_{0}^{\prime} /\left(1-\phi_{0}\right) \in \mathcal{R} \mathcal{V}_{-\kappa_{0}-1}(0)$, with $\kappa_{0} \in[0,+\infty]$, then if furthermore $M \circ T \in \mathcal{R} \mathcal{V}_{\widetilde{\alpha}}(1)$ for some index $\widetilde{\alpha} \in(0,+\infty)$, then

$$
m_{\widetilde{\phi}}=-\widetilde{\phi}^{\prime} /(1-\widetilde{\phi}) \in \mathcal{R} \mathcal{V}_{\widetilde{\kappa}-1}(0) \text { with } \widetilde{\kappa}=\kappa_{0} .
$$

The proof is postponed to Appendix B.

Theorem 3.4 (Transformed case with $\widetilde{r}=0$ ) Let $\phi_{0}$ be an initial generator as in Assumption 1.1 and $T$ be an admissible transformation as in Definition 3.1. Assume that $\psi_{0} \in \mathcal{R} \mathcal{V}_{-r_{0}}(0)$, with $r_{0}=0$ and that $\bar{T} \in \mathcal{R} \mathcal{V}_{\tilde{a}}(0)$, for some index $\widetilde{a} \in(0,+\infty)$. Denote by $\widetilde{\lambda}_{L}^{(h, d-h)}(u)=\frac{\widetilde{\delta}^{(d)}(u)}{\widetilde{\delta}^{(d-h)}(u)}$, with $\widetilde{\delta}^{(d)}$ the transformed diagonal section associated to the d-dimensional copula $\widetilde{C}$ in Equation (6). Then one gets $\widetilde{\lambda}_{L}^{(h, d-h)}=\lim _{u \rightarrow 0^{+}} \widetilde{\lambda}_{L}^{(h, d-h)}(u)=0$.

Assume that $\mu_{\phi_{0}}=-\phi_{0}^{\prime} / \phi_{0} \in \mathcal{R} \mathcal{V}_{k_{0}-1}(\infty)$, with $k_{0} \in[0,+\infty)$. Then

$$
\widetilde{\lambda}_{L}^{(h, d-h)}(u)=\frac{\widetilde{\delta}^{(d)}(u)}{\widetilde{\delta}^{(d-h)}(u)} \in \mathcal{R} \mathcal{V}_{\widetilde{z}}(0), \quad \text { with } \widetilde{z}=d^{k_{0}}-(d-h)^{k_{0}} .
$$

Theorem 3.4 comes down trivially from Theorems 3.3 and 2.2 .

Theorem 3.5 (Transformed case with $\widetilde{\rho}=1$ ) Let $\phi_{0}$ be an initial generator as in Assumption 1.1 and $T$ be an admissible transformation as in Definition 3.1. Assume $\widetilde{\psi} \in \mathcal{R} \mathcal{V}_{\widetilde{\rho}}(1)$, with $\widetilde{\rho}=1$ and that the associated $\widetilde{\phi}$ is a $d$ times continuously differentiable generator. Denote by $\widetilde{\lambda}_{U}^{(h, d-h)}(u)=\frac{\widetilde{r}_{d}(u)}{\widetilde{r}_{d-h}(u)}$, with $\widetilde{r}_{d}(u)=\sum_{i=1}^{d}(-1)^{i} C_{d}^{i} \widetilde{\delta}^{(i)}(u)$ and $\widetilde{\delta}^{(i)}$ the transformed diagonal section associated to the $i$-dimensional copula $\widetilde{C}$ in Equation (6). Then one gets, $\widetilde{\lambda}_{U}^{(h, d-h)}=\lim _{u \rightarrow 1^{-}} \widetilde{\lambda}_{U}^{(h, d-h)}(u)=0$.

Furthermore,

- if $(-D)^{d} \widetilde{\phi}(0)$ is finite and not zero, where $D$ is the derivative operator, then we get

$$
\widetilde{\lambda}_{U}^{(h, d-h)}(u) \in \mathcal{R} \mathcal{V}_{h}(1)
$$

- if $\widetilde{\psi^{\prime}}(1)=0$ and the function $\widetilde{L}(s):=s \frac{\mathrm{d}}{\mathrm{d} s}\left\{\frac{\widetilde{\psi}(1-s)}{s}\right\}$ is positive and $\widetilde{L} \in \mathcal{R} \mathcal{V}_{0}(0)$, then we get

$$
\widetilde{\lambda}_{U}^{(h, d-h)}(u) \in \mathcal{R} \mathcal{V}_{0}(1)
$$

Theorem 3.5 comes down directly by Theorem 2.3 .

Remark 3 Notice that if $r_{0}=0$ in Theorem 3.2 then in particular $\widetilde{\lambda}_{L}^{(h, d-h)}=0$, for all $h$ and $d$. This means for instance that if one considers as initial generator $\psi_{0}(t)=-\ln (t)$, i.e., the independent copula, it is not possible, with this kind of transformations $T \in \mathcal{R} \mathcal{V}_{\widetilde{a}}(0)$ with $\widetilde{a}>0$, to generate some dependency in the multivariate transformed lower tails. 


\subsection{Transformations using conversion functions}

For inferential purposes, it is not always convenient to estimate a distortion $T$ with bounding constraints on $[0,1]$. It can be helpful to define a distortion on the whole set $\mathbb{R}$ and then rescale it in order to ensure that the resulting distortion maps $[0,1]$ into $[0,1]$. In this section, we focus on distortions that are defined by rescaling a particular real function, called conversion function, onto $[0,1]$.

Furthermore, some particular shapes of conversion functions help fitting distorted multivariate distributions (see Bienvenüe and Rullière (2012) in dimension one and Di Bernardino and Rullière (2013b), Di Bernardino and Rullière (2014) in higher dimensions). As an example, it is easy to fit a target function by a piecewise linear one, and thus by a smooth composition of hyperbolas. These particular shapes have asymptotes, and we thus focus here on this special case. We show that this restriction is however sufficient to reach any tail dependence coefficients, and that it helps parameterizing the distortion, since the asymptotes slopes are then given.

We consider transformations $T_{f, G}:[0,1] \rightarrow[0,1]$ such that

$$
T_{f, G}(u)= \begin{cases}0 & \text { if } u=0, \\ G \circ f \circ G^{-1}(u) & \text { if } 0<u<1, \\ 1 & \text { if } u=1,\end{cases}
$$

where $f$ is any continuous bijective increasing function, $f: \mathbb{R} \rightarrow \mathbb{R}$, and is said to be a conversion function. Transformations of this kind are presented in the univariate case in Bienvenüe and Rullière (2011). The transformation $T_{f, G}$ has support $[0,1]$, and the function $G$ aims at transferring this support on the whole real line $\mathbb{R}$, in order to allow $f$ to be defined on the whole set $\mathbb{R}$, without bounding constraints. The function $G$ is thus chosen as a continuous and invertible c.d.f with support $\mathbb{R}$, i.e. such that $\forall x \in \mathbb{R}, G(x) \in(0,1)$. The distribution $G$ is a proper non-defective distribution, so that $\lim _{x \rightarrow-\infty} G(x)=0$ and $\lim _{x \rightarrow \infty} G(x)=1$, where these bounds are never reached due to the chosen support of $G$.

In Assumption 3.1 (resp. 3.2 below, we summarize the assumption setting used in the following, for the study of the transformed multivariate lower (resp. upper) dependence coefficients, using particular transformations $T_{f, G}$ in (11).

Assumption 3.1 (Lower-tails: assumptions on $\left.\phi_{0}, f, G\right)$ Assume that $\phi_{0}, f$ and $G$ are continuous and differentiable functions, strictly monotone with respective proper inverse functions denoted $f^{-1}, \psi_{0}=\phi_{0}^{-1}$ and $G^{-1}$. Furthermore,

i) The function $f$ has an asymptote $\bar{f}(x):=a x+b$ as $x$ tends to $-\infty$, for $a \in(0,+\infty)$ and $b \in(-\infty,+\infty)$.

ii) The inverse initial generator $\psi_{0}$ is regularly varying at 0 with some index $-r_{0}$, that is $\psi_{0} \in \mathcal{R} \mathcal{V}_{-r_{0}}(0)$, with $r_{0} \in[0,+\infty]$.

iii) The function $G$ is a non-defective continuous c.d.f. with support $\mathbb{R}$. The following rate of $G$ is regularly varying with some index $g-1: m_{G}=G^{\prime} / G \in \mathcal{R} \mathcal{V}_{g-1}(-\infty)$, with $g \in(0,+\infty)$.

Assumption 3.2 (Upper-tails: assumptions on $\phi_{0}, f, G$ ) Assume that $\phi_{0}, f$ and $G$ are continuous and differentiable functions, strictly monotone with respective proper inverse functions denoted $f^{-1}, \psi_{0}=\phi_{0}^{-1}$ and $G^{-1}$. Furthermore,

i) The function $f$ has an asymptote $\bar{f}(x):=\alpha x+\beta$ as $x$ tends to $+\infty$, for $\alpha \in(0,+\infty)$ and $\beta \in(-\infty,+\infty)$.

ii) The inverse initial generator $\psi_{0}$ is regularly varying at 1 with some index $\rho_{0}$, i.e., $\psi_{0} \in \mathcal{R} \mathcal{V}_{\rho_{0}}(1)$, with $\rho_{0} \in[1,+\infty]$.

iii) The function $G$ is a non-defective continuous c.d.f. with support $\mathbb{R}$. The hazard rate of $G$ is regularly varying with some index $\gamma-1$, that is $\mu_{G}=G^{\prime} / \bar{G} \in \mathcal{R} \mathcal{V}_{\gamma-1}(\infty)$, with $\bar{G}=1-G$ and $\gamma \in(0,+\infty)$.

Under Assumptions 3.1 and 3.2 one can provide the regularly varying property for the considered transformation $T_{f, G}$.

Theorem 3.6 (Regular Variation of transformation $T_{f, G}$ ) Under lower regular Assumption 3.1, we get

$$
T_{f, G} \in \mathcal{R} \mathcal{V}_{\widetilde{a}}(0) \text {, with } \widetilde{a}=a^{g} .
$$

Under upper regular Assumption 3.2, we get

$$
M \circ T_{f, G} \in \mathcal{R} \mathcal{V}_{\widetilde{\alpha}}(1), \text { with } \widetilde{\alpha}=\alpha^{\gamma} .
$$


The proof is postponed to Appendix B. Remark that from Theorem 3.6, if $T_{f, G}$ satisfies Assumption 3.1, then $T \in \mathcal{R} \mathcal{V}_{\widetilde{a}}(0)$ for some index $\widetilde{a} \in(0,+\infty)$ as required in Theorem 3.4 .

Under Assumptions 3.1 and 3.2 the following result characterizes the regularly varying property for the inverse transformed generator $\psi=T_{f, G} \circ \phi_{0}$ and the multivariate tail dependence coefficients associated to the transformed Archimedean copula $\widetilde{C}_{T_{f, G}, C_{0}}$, with $T_{f, G}$ as in Equation (11).

Corollary 3.1 Let $\phi_{0}$ be an initial generator as in Assumption 1.1 and $T_{f, G}$ be an admissible transformation as in Definition 3.1. Assume that $\phi_{0}, f$ and $G$ satisfy Lower-tail Assumptions 3.1. Then

i) the inverse transformed generator $\widetilde{\psi}$ is such that

$$
\widetilde{\psi} \in \mathcal{R} \mathcal{V}_{-\widetilde{r}}(0) \text {, with } \widetilde{r}=r_{0} \cdot a^{-g}
$$

ii) the associated transformed lower multivariate lower tail dependence coefficient is given by:

$$
\widetilde{\lambda}_{L}^{(h, d-h)}= \begin{cases}\text { see Theorem 3.4 } & \text { if } \widetilde{r}=0, \\ d^{-a^{g} r_{0}^{-1}(d-h)^{a^{g}} r_{0}^{-1},}, & \text { if } \widetilde{r} \in(0,+\infty), \\ 1, & \text { if } \widetilde{r}=+\infty\end{cases}
$$

Assume now that $\phi_{0}, f$ and $G$ satisfy Upper-tail Assumption 3.2. Then

i) the inverse transformed generator $\widetilde{\psi}$ is such that

$$
\widetilde{\psi} \in \mathcal{R} \mathcal{V}_{\widetilde{\rho}}(1), \text { with } \widetilde{\rho}=\rho_{0} \cdot \alpha^{-\gamma} \quad \text { and } \quad \widetilde{\rho} \in[1,+\infty]
$$

ii) the associated transformed upper multivariate lower tail dependence coefficient is given by:

$$
\widetilde{\lambda}_{U}^{(h, d-h)}= \begin{cases}\text { see Theorem 3.5, } & \text { if } \widetilde{\rho}=1, \\ \frac{\sum_{i=1}^{d} C_{d}^{i}(-1)^{i} \cdot i^{\alpha^{\gamma}} \rho_{0}^{-1}}{\sum_{i=1}^{d-h} C_{d-h}^{i}(-1)^{i} \cdot i^{\alpha \gamma} \rho_{0}^{-1}}, & \text { if } \widetilde{\rho} \in(1,+\infty), \\ 1, & \text { if } \widetilde{\rho}=+\infty .\end{cases}
$$

Corollary 3.1 comes down directly by applying Theorems 3.1 and 3.2 with $\widetilde{a}=a^{g}$ and $\widetilde{\alpha}=\alpha^{\gamma}$ respectively. Other direct applications of Theorem 3.3 involving hazard rates variation indexes $k$ and $\kappa$ could be proposed, which can be useful for the asymptotic independence case, as in Theorem 2.2. or for the behavior of the transformed diagonal section, as in Lemma 2.1.

Remark 4 From Corollary 3.1, as $\widetilde{r}=r_{0} \cdot a^{-g} \in[0,+\infty]$ and $\widetilde{\rho}=\rho_{0} \cdot \alpha^{-\gamma} \in[1,+\infty]$, an implicit admissible range for the positive slope parameters $a$ and $\alpha$ is

$$
a \in(0, \infty) \quad \text { and } \quad \alpha \in\left(0, \rho_{0}^{1 / \gamma}\right]
$$

As one can see in previous Corollary 3.1, the impact of initial generator $\phi_{0}$, conversion function $f$ and distribution $G$ are clearly separated via respective coefficients couples $\left(r_{0}, \rho_{0}\right),(a, \alpha),(g, \gamma)$. It is thus possible to modify any of these parts of the global distortion $T_{f, G}=G \circ f \circ G^{-1}$ to fit a desired tail dependence.

Remark 5 From Corollary 3.1, we know that if $\psi_{0} \in \mathcal{R} \mathcal{V}_{-r_{0}}(0)$, with $r_{0}=0$, then $\widetilde{r}=r_{0} \cdot a^{-g}=0$ for all a and $g$ (see Equation (12)). Then automatically $\widetilde{\lambda}_{L}^{(h, d-h)}=0$ (see Theorem 3.4. with $\widetilde{a}=a^{g}$ ). Conversely in the upper case $\widetilde{\rho}=\rho_{0} \cdot \alpha^{-\gamma}$ (see Equation (14) ) then $\rho_{0}=1$ does not guarantee in Theorem 3.5 that the transformed copula is also asymptotic independent in the upper tails, i.e., $\widetilde{\rho}=1$ (see also Theorem 3.2).

In the following we provide we provide an easy way to construct the rescaling distribution $G$ such that $m_{G}=G^{\prime} / G \in$ $\mathcal{R} \mathcal{V}_{g-1}(-\infty)$, with $g \in(0,+\infty)$ (see Lower-tail Assumption 3.1) or $\mu_{G}=G^{\prime} / \bar{G} \in \mathcal{R} \mathcal{V}_{\gamma-1}(\infty)$ with $\gamma \in(0,+\infty)$ (see Upper-tail Assumption 3.2 . 
Lemma 3.1 Let $F$ and $G$ be two non-defective cumulative distribution functions with support $\mathbb{R}$, with proper inverse functions, and let $P$ be an increasing differentiable bijection from $\mathbb{R}$ to $\mathbb{R}$. If $G=F \circ P$ then the following relation holds between respective hazard rates of $F$ and $G$ :

$$
m_{G}=m_{F} \cdot P^{\prime} \quad \text { and } \quad \mu_{G}=\mu_{F} \cdot P^{\prime},
$$

where $m_{F}=F^{\prime} / F, m_{G}=G^{\prime} / G$ and $\mu_{F}=F^{\prime} / \bar{F}, \mu_{G}=G^{\prime} / \bar{G}$. In particular, let

$$
P_{g, \gamma}(x)= \begin{cases}-|x|^{g}, & x<0 \\ +|x|^{\gamma}, & x \geq 0\end{cases}
$$

with $\gamma, g \in(0,+\infty)$ and $(g-1)(\gamma-1) \geq 0$, so that $\lim _{x \rightarrow 0^{-}} P_{g, \gamma}^{\prime}(x)=\lim _{x \rightarrow 0^{+}} P_{g, \gamma}^{\prime}(x)$. If $m_{F} \in \mathcal{R} \mathcal{V}_{g_{0}}(-\infty)$ and $\mu_{F} \in \mathcal{R} \mathcal{V}_{\gamma_{0}}(\infty)$, then

$$
m_{G} \in \mathcal{R} \mathcal{V}_{g_{0}+g-1}(-\infty) \quad \text { and } \quad \mu_{G} \in \mathcal{R} \mathcal{V}_{\gamma_{0}+\gamma-1}(\infty)
$$

If furthermore $g_{0}+g \in(0,+\infty)$ and $\gamma_{0}+\gamma \in(0,+\infty)$, since $G$ is by assumption a valid distribution, then $G$ satisfies the point iii) in respective Assumptions 3.1 and 3.2 .

The proof is postponed to Appendix B. It would be possible to extend the assumptions of Lemma 3.1. However in Example 1 below, we focus on distributions that are defined on the whole support $\mathbb{R}$, readily invertible and constructed by using the function $P_{g, \gamma}$. Parameters $g$ and $\gamma$ must be positive in order to get an increasing cdf for the induced distribution $G$.

It is worth mentioning that for distributions $F$ having a symmetric density, such that $F^{\prime}(-x)=F^{\prime}(x)$, for all $x \in \mathbb{R}$, one easily shows that $m_{F}(-x)=\mu_{F}(x)$, for all $x \in \mathbb{R}$. In this symmetric case, when the hazard rate is regularly varying, both rates $m_{F}=F^{\prime} / F$ and $\mu_{F}=F^{\prime} / \bar{F}$ have the same respective regular variation index at $-\infty$ and $+\infty$. Using the idea of Lemma 3.1. we now present two particular distributions, defined on the whole real line, with regularly varying hazard rates. In particular, as explained in the beginning of Section 3.1, we exclude distributions having support $\left[x_{0}, \infty\right)$ or $\left(-\infty, x_{0}\right]$ for some finite given real $x_{0}$.

Example 1 (Examples of rescaling distribution $G$ ) Define $P_{g, \gamma}$ as in Lemma 3.1, with $g \in(0, \infty), \gamma \in$ $(0, \infty)$, and $(g-1)(\gamma-1) \geq 0$. Define $R(x)=\frac{x-\mu}{\sigma}, \mu \in \mathbb{R}, \sigma \in(0, \infty)$, and $G=F \circ P_{g, \gamma} \circ R$.

- Considering $F(x)=\operatorname{logit}^{-1}(x), F$ has a symmetric density and a slowly varying hazard rate,

$$
m_{F} \in \mathcal{R} \mathcal{V}_{g_{0}}(-\infty) \quad \text { and } \quad \mu_{F} \in \mathcal{R} \mathcal{V}_{\gamma_{0}}(+\infty) \text {, with } g_{0}=\gamma_{0}=0
$$

One easily shows that the rates $m_{G}=G^{\prime} / G$ and $\mu_{G}=G^{\prime} / \bar{G}$ are such that

$$
m_{G} \in \mathcal{R} \mathcal{V}_{g-1}(-\infty) \quad \text { and } \quad \mu_{G} \in \mathcal{R} \mathcal{V}_{\gamma-1}(\infty)
$$

This power-logit case gives an example of a function $G$ that can exhibit any chosen coefficient $g, \gamma \in(0,+\infty)$, thus satisfying both Assumptions 3.1 and 3.2 .

- Considering $F(x)=\exp (-\exp (-x)), F$ has a lower rate $m_{F}$ rapidly varying at $-\infty$ (denoted by $m_{F} \in$ $\mathcal{R} \mathcal{V}_{\infty}(-\infty)$; see Section 2.4 in Bingham et al. (1989) for a formal definition of rapid variation), and a slowly varying upper hazard rate $\mu_{F}$,

$$
m_{F} \in \mathcal{R} \mathcal{V}_{\infty}(-\infty) \text {, with } g_{0}=+\infty \quad \text { and } \quad \mu_{F} \in \mathcal{R} \mathcal{V}_{\gamma_{0}}(+\infty) \text {, with } \gamma_{0}=0
$$

One easily shows that the rates $m_{G}=G^{\prime} / G$ and $\mu_{G}=G^{\prime} / \bar{G}$ are such that

$$
m_{G} \in \mathcal{R} \mathcal{V}_{\infty}(-\infty) \quad \text { and } \quad \mu_{G} \in \mathcal{R} \mathcal{V}_{\gamma-1}(\infty)
$$

This power-Gumbel case gives an example of a function $G$ that can exhibit any chosen coefficient $\gamma \in(0,+\infty)$ but where $m_{G} \in \mathcal{R} \mathcal{V}_{\infty}(-\infty)$, thus satisfying Assumption 3.2 but not Assumption 3.1. Using such a powerGumbel distribution would require a separated treatment for the lower tail case.

Remark that many other distributions having regularly varying hazard rate can be proposed. For instance, a study of some distributions having regularly varying hazard rate is given in Asmussen and Kortschak (2013). Interesting relations can be found between the class of subexponential distributions (denoted by $\mathcal{S}$ ) and regularly varying 
hazard rates. The interested reader is referred for instance to Klüppelberg (1989a), Klüppelberg (1989b), Su and Tang (2003). Recall that subexponential distributions have unbounded support and may belong to Fréchet and Gumbel maximum domain of attraction (see Section 4 in Goldie and Klüppelberg (1998)). In particular Goldie and Klüppelberg (1998) proved that if $\lim _{x \rightarrow \infty} \mu_{G}(x)=\lim _{x \rightarrow \infty} \frac{G^{\prime}(x)}{\bar{G}(x)}=0, \lim _{x \rightarrow \infty} x \mu_{G}(x)=\infty$, and $\mu_{G} \in R V_{\gamma-1}(\infty)$, with $\gamma \in(0,1)$, then $G \in \mathcal{S}^{*}$, where "the class $\mathcal{S}^{*}$ is almost $\mathcal{S} \cap\{G: \mathbb{E}[G]<\infty\}$." A precise formulation can be found in Klüppelberg (1988) (see Remark 1 and Corollary 3.9 in Goldie and Klüppelberg (1998)). Remark that this type of distribution functions above satisfies Assumption 3.2 .

\section{Transformed copulas with given tails}

\subsection{Hyperbolic conversion functions and tail dependence}

In practice, one can estimate lower and upper tail coefficients. A large literature has been developed in order to propose consistent non-parametric tail dependence coefficients estimators, essentially based on the non-parametric copula estimator and Extreme Value Theory (see for instance de Haan and Ferreira (2006), Schmidt and Stadtmüller (2006), Dobric and Schmid (2005)).

However, even when such tail coefficients are perfectly known, using usual uni-parametric Archimedean generators does not allow to get both lower and upper tail coefficients in the general case where their values belong to $(0,1)$. Indeed, commonly used families of Archimedean copulas have the property that either $\lambda_{U}=\lambda_{L}$ or that only one of the coefficients is nonzero. For example, in Table 1 of Charpentier and Segers (2007), only two uni-parametric generators among 23 exhibit both lower tail dependence and upper tail dependence (see generators numbered 12 and 14 in their table). Unfortunately none of these two allow to choose separately lower and upper tail coefficients as they both depend on one single parameter. In this sense, Larsson and Nešlehová (2011) provided an example in order to overcome this restrictive behavior of classical Archimedean copulas (see Example 2 in Larsson and Nešlehová (2011)). Even for other kinds of copulas, like elliptical copulas, it is challenging to find simple parametric expressions with both lower and upper tail dependencies that are parametrized by more than one parameter. There exist some two-parameter copulas that have both upper and lower tail dependence, as for example, the BB1, BB4 and BB7 copulas in Joe (1997b). Another method to generate copulas that meet the requirement is to use compositions of two Laplace transforms, with one from a one-parameter family and the other from another one-parameter family. However, as stated in Larsson and Nešlehová (2011), it is rather difficult to construct new Archimedean copula models that exhibit specific tail behavior.

Here we propose a generic way to construct families of Archimedean generators presenting a chosen couple of lower and upper tail coefficients. This construction relies directly on theoretical results of the previous Section 3 . One advantage of such a construction is that it can be easily adapted to data by adding parameters without changing targeted lower and upper tail coefficients (for this type of construction see Di Bernardino and Rullière (2013a), Di Bernardino and Rullière (2014)).

Under Assumptions 3.1 and 3.2 the transformation $T_{f, G}=G \circ f \circ G^{-1}$ is a transformation with a particular conversion function $f$ having asymptotes at $-\infty$ or $+\infty$. This is exactly the framework of Di Bernardino and Rullière (2013a), Di Bernardino and Rullière (2013b) and Di Bernardino and Rullière (2014) where conversion functions $f$ are compositions of hyperbolas, with asymptotes at both $-\infty$ or $+\infty$. In these papers, slopes $a$ and $\alpha$ of each asymptote of $f$ are tractable given functions of some isolated parameters of $f$. Parametric estimation of copulas within this framework is thus possible following the methodology of these articles, even with given tail coefficients. The only change is that some parameters will be fixed to some values instead of being estimated.

Consider for example a transformed Archimedean copula, having generator $\widetilde{\phi}=T_{f, G} \circ \phi_{0}$, where $\phi_{0}$ is an initial given generator, and where the transformation is as in Equation (11), with in particular for any $x \in(0,1)$,

$$
T_{f, G}(x)=G \circ f \circ G^{-1}(x) .
$$

Choose for example a simple hyperbolic conversion function $f(x)=H(x), x \in \mathbb{R}$, as defined in Bienvenüe and Rullière (2011), with

$$
H_{m, h, p_{1}, p_{2}, \eta}(x)=m-h+\left(\mathrm{e}^{p_{1}}+\mathrm{e}^{p_{2}}\right) \frac{x-m-h}{2}-\left(\mathrm{e}^{p_{1}}-\mathrm{e}^{p_{2}}\right) \sqrt{\left(\frac{x-m-h}{2}\right)^{2}+\mathrm{e}^{\eta-\frac{p_{1}+p_{2}}{2}}},
$$

with $m, h, p_{1}, p_{2} \in \mathbb{R}$, and one smoothing parameter $\eta \in \mathbb{R}$. Function $H$ has been chosen in order to have unbounded real parameters, and to be readily invertible: a simple change of the sign of some parameters leads to the inverse 
function. After some calculations, one can check indeed that

$$
H_{m, h, p_{1}, p_{2}, \eta}^{-1}(x)=H_{m,-h,-p_{1},-p_{2}, \eta}(x) .
$$

As a consequence, transformations $T_{H, G}$ based on conversion functions $H$ will also be readily invertible since $\left(T_{H, G}\right)^{-1}=T_{H^{-1}, G}$.

When the smoothing parameter $\eta$ tends to $-\infty$, the hyperbole $H$ tends to the angle function

$$
A_{m, h, p_{1}, p_{2}}(x)=m-h+(x-m-h)\left(\mathrm{e}^{p_{1}} 1_{\{x<m+h\}}+\mathrm{e}^{p_{2}} 1_{\{x>m+h\}}\right) .
$$

It is clear that in this framework, $f=H$ satisfies respective Assumptions 3.1 and 3.2. In particular, $f$ has an asymptote $a x+b$ at $-\infty$ with $a=\mathrm{e}^{p_{1}}$, and an asymptote $\alpha x+\beta$ at $+\infty$ with $\alpha=\mathrm{e}^{p_{2}}$. More complex conversion functions could be used by composing such hyperbolas (see Bienvenüe and Rullière (2011) and Di Bernardino and Rullière (2014)). For the sake of clarity, we consider here a single hyperbola.

Assume that the inverse initial generator $\psi_{0}$ satisfies Assumptions 3.1 and 3.2 with in particular $\psi_{0} \in \mathcal{R} \mathcal{V}_{-r_{0}}(0)$, with $r_{0} \in(0,+\infty)$, and $\psi_{0} \in \mathcal{R} \mathcal{V}_{\rho_{0}}(1)$, with $\rho_{0} \in[1,+\infty)$. Assume that the distribution $G$ also satisfies Assumptions 3.1 and 3.2 , with in particular $m_{G}=G^{\prime} / G \in \mathcal{R} \mathcal{V}_{g-1}(-\infty)$, with $g \in(0,+\infty)$ and $\mu_{G}=G^{\prime} / \bar{G} \in \mathcal{R} \mathcal{V}_{\gamma-1}(\infty)$, where $G=1-G$ and $\gamma \in(0,+\infty)$. For example, if one set $G=\operatorname{logit}^{-1}$, then $g=\gamma=1$.

From Corollary 3.1, the transformed multivariate tail coefficients can be written as:

$$
\widetilde{\lambda}_{L}^{(h, d-h)}=d^{-a^{g} r_{0}^{-1}}(d-h)^{a^{g} r_{0}^{-1}} \quad \text { and } \quad \widetilde{\lambda}_{U}^{(h, d-h)}=\frac{\sum_{i=1}^{d} C_{d}^{i}(-1)^{i} \cdot i^{\alpha^{\gamma} \rho_{0}^{-1}}}{\sum_{i=1}^{d-h} C_{d-h}^{i}(-1)^{i} \cdot i^{\alpha^{\gamma} \rho_{0}^{-1}}} .
$$

Let us illustrate the bivariate case, for which expressing $a$ and $\alpha$ as functions of $\widetilde{\lambda}_{L}^{(h, d-h)}$ and $\widetilde{\lambda}_{U}^{(h, d-h)}$ is straightforward. In the bivariate version of Corollary 3.1. $\widetilde{\lambda}_{L}^{(1,1)}=2^{-a^{g} r_{0}^{-1}}$ and $\widetilde{\lambda}_{U}^{(1,1)}=2-2^{\alpha^{\gamma} \cdot \rho_{0}^{-1}}$, so that if these tail coefficients are given, then we can easily find $a=\mathrm{e}^{p_{1}}$ and $\alpha=\mathrm{e}^{p_{2}}$ as functions of $\widetilde{\lambda}_{L}^{(1,1)}$ and $\tilde{\lambda}_{U}^{(1,1)}$. One can check that with the chosen assumptions, $\widetilde{r}=r_{0} a^{-g} \in(0, \infty)$ so that $\widetilde{\lambda}_{L}^{(1,1)} \in(0,1)$. For the upper tail, $\widetilde{\rho}=\rho_{0} \alpha^{-\gamma}$ must belong to $[1, \infty]$ if the transformed generator is valid, and due to chosen assumptions, $\widetilde{\rho}<+\infty$ so that $\widetilde{\lambda}_{U}^{(1,1)} \in[0,1)$. We get in this case

$$
p_{1}=\frac{1}{g} \ln \left(-r_{0} \frac{\ln \widetilde{\lambda}_{L}^{(1,1)}}{\ln 2}\right) \quad \text { and } \quad p_{2}=\frac{1}{\gamma} \ln \left(\rho_{0} \frac{\ln \left(2-\widetilde{\lambda}_{U}^{(1,1)}\right)}{\ln 2}\right) .
$$

Finally, only $m, h$ and $\eta$ remain to be estimated and the detailed methodology of Di Bernardino and Rullière (2014) can be adapted to this purpose. For the sake of simplicity, we have illustrated here the case where $f$ is a single hyperbola, in the dimension $d=2$, when we focus in intermediate cases for lower and upper tail dependence, excluding perfect tail dependence or perfect tail independence. One can easily consider composite hyperbolas, with more parameters and the ability to fit both the tails and the central part of the copula. One can also consider the multivariate case, or special case of non intermediate tail dependence, for example when $r_{0}=0$, when $\widetilde{\lambda}_{L}^{(1,1)}=0$, or when $\tilde{\lambda}_{U}^{(1,1)}=1$. Other interesting cases are the tail behaviour when $G$ is rapidly varying, as for lower tail dependence when $G$ is Gumbel distributed, i.e., $G(x)=\exp (-\exp (-x))$ (see Section 2.4 in Bingham et al. (1989)). These extensions would be out of the scope of the present section, which just illustrates the methodology given tail dependence coefficients. Note that for one single hyperbola, this gives a class of Archimedean copulas exhibiting given (estimated) upper and lower tail dependence coefficients.

\subsection{Illustration with logit transformations}

In the following we provide an illustration of the procedure described above. Let the initial copula $C_{0}$ be a Clayton copula with parameter $\theta>0$ with $\psi_{0}(t)=\frac{1}{\theta}\left(t^{-\theta}-1\right)$. Assume that we want to transform the tails of the bivariate distribution $(X, Y)$ in order to obtain an arbitrarily chosen couple of target tail coefficients: $\widetilde{\lambda}_{L}^{(1,1)}=1 / 4$ and $\tilde{\lambda}_{U}^{(1,1)}=3 / 4$.

Then the transformed generator is such that $\widetilde{\psi}=\psi_{0} \circ T_{f, G}^{-1}$, where $T_{f, G}^{-1}=G \circ H^{-1} \circ G^{-1}$ (see Equation (17)). In the following, we consider $G=\operatorname{logit}^{-1}$, then $g=\gamma=1$. Using Equation 18 we can obtain the parameters $p_{1}$ and $p_{2}$ of the conversion function $H$ given in (16).

In Table 1 we summarize the parameters setting considered. In particular we take arbitrarily chosen different values of parameters $m, h, \eta$ and $\theta$, in order to illustrate the variety of generators that can exhibit the same tail dependence 


\begin{tabular}{|c|c|c|c|c|c|c|c|c|c|c|}
\hline & \multicolumn{4}{|c|}{ chosen parameters } & \multicolumn{3}{c|}{ deduced parameters } & \multicolumn{4}{|c|}{ tails coefficients } \\
\hline & $m$ & $h$ & $\eta$ & $\theta$ & $p_{1}$ & $p_{2}$ & $\lambda_{0, L}^{(1,1)}$ & $\lambda_{0, U}^{(1,1)}$ & $\widetilde{\lambda}_{L}^{(1,1)}$ & $\widetilde{\lambda}_{U}^{(1,1)}$ \\
\hline $\mathrm{A}$ & 0.5 & 0.9 & -1 & 2 & 1.386 & -1.133 & 0.707 & 0 & 0.25 & 0.75 \\
\hline $\mathrm{B}$ & 0.5 & -0.9 & -1 & 2 & 1.386 & -1.133 & 0.707 & 0 & 0.25 & 0.75 \\
\hline $\mathrm{C}$ & 0.5 & 0.9 & -2 & 4 & 2.079 & -1.133 & 0.841 & 0 & 0.25 & 0.75 \\
\hline $\mathrm{D}$ & 2 & -0.9 & 1 & 0.2 & -0.916 & -1.133 & 0.031 & 0 & 0.25 & 0.75 \\
\hline
\end{tabular}

Table 1: Considered parameters settings.

coefficients. Only $p_{1}$ and $p_{2}$ are deduced from chosen values $\widetilde{\lambda}_{L}^{(1,1)}=1 / 4$ and $\widetilde{\lambda}_{U}^{(1,1)}=3 / 4$ using Equation (18). The set of parameters $m, h, p_{1}, p_{2}, \eta$ fully characterize the transformation $T_{f, G}$, and $\theta$ characterize the initial nontransformed copula $C_{0}$. Then the transformed copula $\widetilde{C}_{T_{f, G}, C_{0}}$ is fully characterized by the parameters in Table 1 .

First, one can check that proposed transformed generators are valid Archimedean generators. By construction, $\widetilde{\psi}$ is an increasing transformation of a decreasing initial generator, so that $\widetilde{\psi}$ is a positive decreasing function, with $\widetilde{\psi}(1)=0$. To be valid, $\widetilde{\psi}$ must be a $d$-monotone function (see McNeil and Nešlehová (2009)), i.e. here a convex function. For all cases presented in Table 1 , we check that the derivatives of $\tilde{\psi}(t)$ are increasing functions of $t$, thus leading to a valid convex generator. The increasing derivatives of $\widetilde{\psi}(t)$ are illustrated in Figure 1 .

By construction, the regular variation indexes $\widetilde{r}$ and $\widetilde{\rho}$ are (see Theorem 2.1 for $d=2$ and $h=1$ ):

$$
\widetilde{r}=-\frac{\ln 2}{\ln \left(\widetilde{\lambda}_{L}^{(1,1)}\right)}=0.5 \quad \text { and } \quad \widetilde{\rho}-1=\frac{\ln 2}{\ln \left(2-\widetilde{\lambda}_{U}^{(1,1)}\right)}-1 \simeq 2.1
$$

These illustrations show that one can propose many generators with given tail dependence coefficients. When fitting some data, it is thus possible to propose a fit that respects some estimated tail dependence coefficients, by deducing parameters $p_{1}$ and $p_{2}$ from tail coefficients and by estimating other parameters $m, h, \eta, \theta$. It is also possible to improve the global quality of the fit by adding some parameters: this can be done easily by compositing more than one hyperbola, as detailed in Di Bernardino and Rullière (2014) for example.

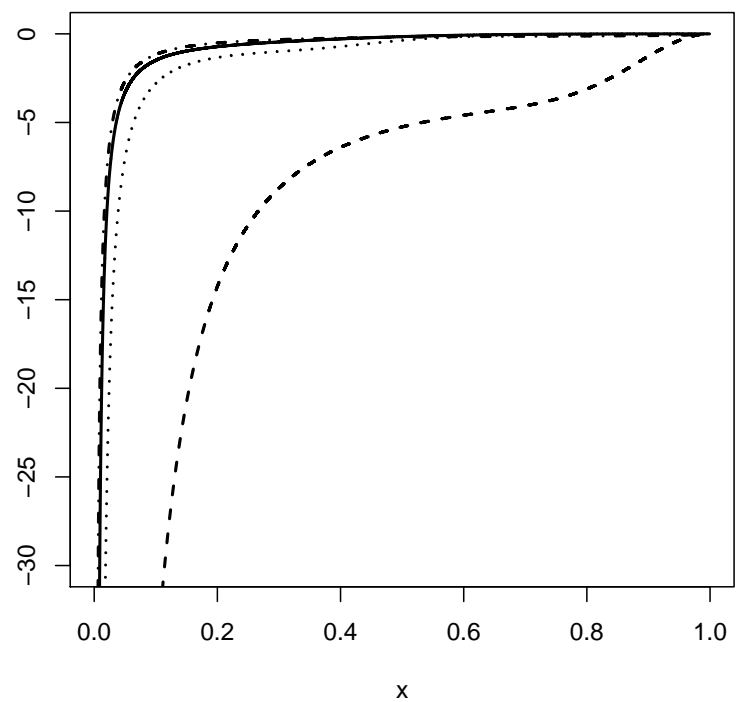

Figure 1: Derivatives of $\widetilde{\psi}$ obtained using the parameter setting gathered in Table 1. Full line corresponds to parameters A in Table 1; dashed line to B; dotted line to C; dashed-dotted line to D.

Recall that in the bivariate case, for a given copula $C$, cdf of a random vector $\left(U_{1}, U_{2}\right)$, one defines lower and upper tail dependence functions for $u \in(0,1)$ as

$$
\lambda_{L}(u)=\mathbb{P}\left[U_{2}<u \mid U_{1}<u\right] \quad \text { and } \quad \lambda_{U}(u)=\mathbb{P}\left[U_{2}>u \mid U_{1}>u\right] .
$$




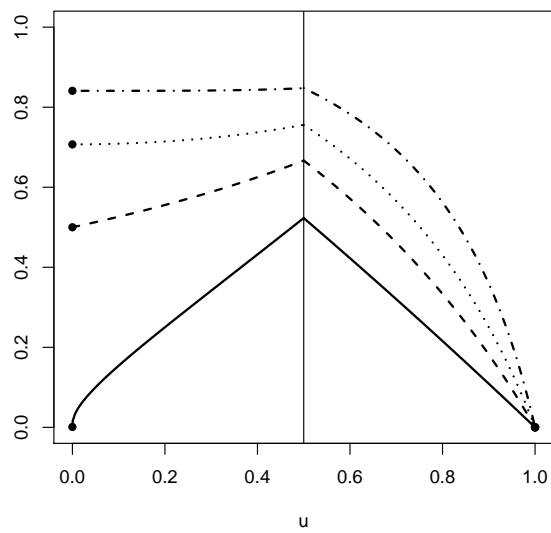

Gumbel copulas

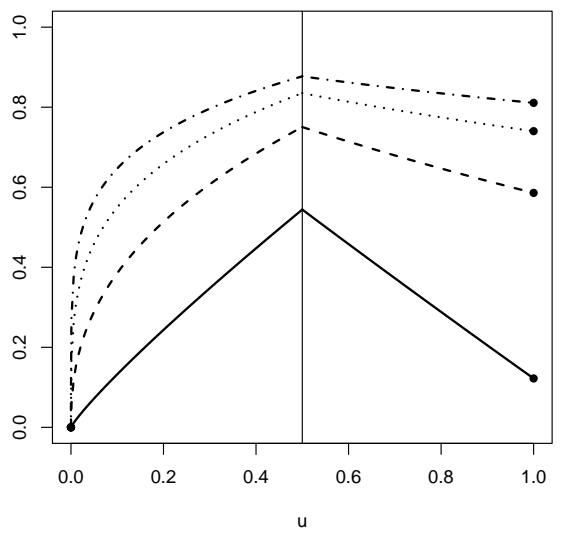

Models A, B, C, D

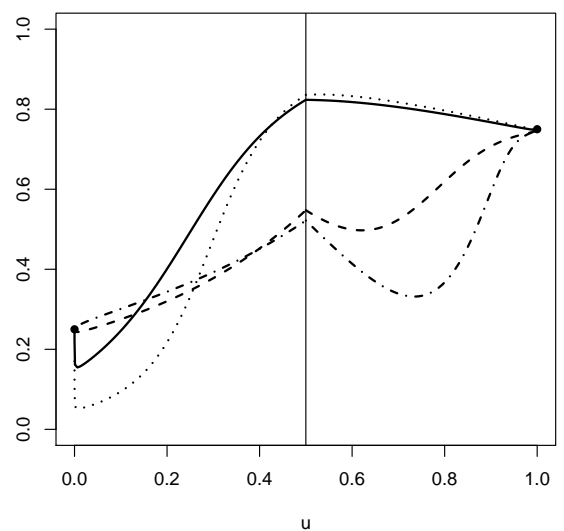

Figure 2: Shape of the tail concentration function $\lambda_{L U}(u)=\mathbf{1}_{\{u \leq 1 / 2\}} \lambda_{L}(u)+\mathbf{1}_{\{u>1 / 2\}} \lambda_{U}(u)$ for some Clayton copulas (left panel), Gumbel copulas (center panel), and for models A,B,C,D in Table 1 (right panel). In Clayton case (left panel) the full line corresponds to $\theta=0.1$, dashed line to $\theta=1$, dotted line to $\theta=2$ and dashed-dotted line to $\theta=4$. In Gumbel case (central panel) the full line corresponds to $\theta=1.1$, dashed line to $\theta=2$, dotted line to $\theta=3$ and dashed-dotted line to $\theta=4$. In the right panel, full line corresponds to parameters $\mathrm{A}$ in Table 1 dashed line to $\mathrm{B}$; dotted line to $\mathrm{C}$; dashed-dotted line to $\mathrm{D}$.

In Figure 2, we have drawn the tail concentration function $\lambda_{L U}(u)=\mathbf{1}_{\{u \leq 1 / 2\}} \lambda_{L}(u)+\mathbf{1}_{\{u>1 / 2\}} \lambda_{U}(u)$ (see for instance Venter (2001) and Durante et al. (2014)) for some Clayton and Gumbel copulas (left and center panel), and for considered models A, B, C, D in Table 1 (right panel). One can notice that left limit at 0 and right limit at 1 of the function $\lambda_{L U}$ gives the respective lower and upper tail dependence coefficients. One retrieves here the chosen tail coefficient targets $1 / 4$ and $3 / 4$ for models A, B, C, D (black dots in right panel). Furthermore, one can see that among models A, B, C, D, in Table1 the ways of converging toward fixed tail coefficients are very different. As noticed in the bivariate case by Avérous and Dortet-Bernadet (2004), "many of the most commonly used parametric families of Archimedean copulas (such as the Clayton, Gumbel, Frank or Ali-Mikhail-Haq systems) possess strong dependence properties: they have the SI or the SD property and are ordered at least by <LTD", where Stochastic Increasingness (SI), Stochastically Decreasingness (SD) and Left-Tail Decreasingness (LTD) definitions are recalled in Avérous and Dortet-Bernadet (2004), Joe (1997a). It implies in particular that for many classical Archimedean copulas (and in particular for Clayton, Gumbel, Frank or Ali-Mikhail-Haq copulas) $\lambda_{L U}$ is nondecreasing on $(0,1 / 2)$ and non-increasing on $(1 / 2,1)$. This can be seen on the left and center panel of Figure 2 , but this is obviously not the case here in the right panel for models A, B, C, D. This surprising shape of $\lambda_{L U}$ function emphasis the large diversity of tail behaviours that can be reached by proposed transformed Archimedean copulas.

\section{$5 \quad$ Further applications}

In this section we propose some illustrations of possible applications of results developed above in this paper. In Section 5.1. using the results recently provided by Larsson and Nešlehová (2011), we analyse the extremal behaviour of the radial part of a transformed copula. Finally in Section 5.2 we analyse the ergodic property of the stationary Markov chain $\left\{U_{t}: t \in \mathbb{Z}\right\}$ with transformed joint distribution $C$ of $\left(U_{0}, U_{1}\right)$.

\subsection{Extremal behaviour of the radial part of a transformed copula}

As shown by McNeil and Nešlehová (2009) (see their Proposition 4.4), if $\mathbf{U}=\left(U_{1}, \ldots, U_{d}\right)$ is a vector distributed according to a $d$-dimensional Archimedian copula with generator $\phi$ as in Equation (2), with generalized inverse generator $\psi=\phi^{-1}$, then

$$
\left(\psi\left(U_{1}\right), \ldots, \psi\left(U_{d}\right)\right) \stackrel{d}{=} R \mathbf{S},
$$

where $\mathbf{S}=\left(S_{1}, \ldots, S_{d}\right)$ is uniformly distributed on the unit simplex $\left\{\mathbf{x} \geq 0 \mid \sum_{k=1}^{d} x_{k}=1\right\}$ and $R$ is an independent non-negative random variable on $[0,+\infty)$ which can be interpreted as the radial part of $\left(\psi\left(U_{1}\right), \ldots, \psi\left(U_{d}\right)\right)$ since $\sum_{k=1}^{d} S_{k}=1$. 
The random vector $\mathbf{S}$ follows a symmetric Dirichlet distribution whereas the distribution of $R \stackrel{d}{=} \sum_{k=1}^{d} \psi\left(U_{k}\right)$ is directly related to the generator $\phi$ through its inverse Williamson transform. Then, the radial part $R$ of the stochastic representation in 19 is directly related to the generator $\phi$ and the probability integral transformation of $\mathbf{U}$, that is,

$$
R \stackrel{d}{=} \psi(C(\mathbf{U})) .
$$

Let $F_{R}$ denote the distribution function of the radial part $R$ in (19). Larsson and Nešlehová (2011) study under which conditions, $F_{R}$ belongs in the maximum domain of attraction of an extreme value distribution, i.e., $F_{R} \in \mathrm{MDA}(H)$, where $H$ is either the Fréchet $\left(\Phi_{\alpha}\right)$, Gumbel $(\Lambda)$ or Weibull $\left(\Psi_{\alpha}\right)$ extreme value type distribution with tail parameter $\alpha>0$. It appears that there is a strong link between tail dependence and attraction domain of $R$ or $1 / R$.

They prove that, for any $\alpha>0$ :

(i) $F_{R} \in \operatorname{MDA}\left(\Phi_{\alpha}\right)$ if and only if $\phi \in \mathcal{R} \mathcal{V}_{-\alpha}(\infty)$,

(ii) $F_{R} \in \operatorname{MDA}\left(\Psi_{\alpha}\right)$ if and only if $\phi \in \mathcal{R} \mathcal{V}_{-\alpha-d+1}\left(x_{F}\right), x_{F} \in(0, \infty)$,

(iii) $F_{R} \in \operatorname{MDA}(\Lambda)$ if and only if $\phi \in \Delta$, where the non-increasing and continuous generator $\phi \in \Delta$ if there exists a measurable function $a: \mathbb{R} \rightarrow(0, \infty)$ such that $\lim _{x \rightarrow x_{F}} \frac{\phi(x+a(x) t)}{\phi(x)}=e^{-t}, t \in \mathbb{R}$,

(see Theorem 1 and Proposition 1 in Larsson and Nešlehová (2011)). Remark that $\phi \in \mathcal{R} \mathcal{V}_{\alpha}\left(x_{F}\right) \Leftrightarrow \phi \circ M_{x_{F}} \circ I \in$ $\mathcal{R} \mathcal{V}_{\alpha}(\infty)$, with $M_{x_{F}}(x)=x_{F}-x$ and $I(x)=1 / x$. The threshold $x_{F}$ is $x_{F}=\sup \left\{x \in \mathbb{R}: F_{R}(x)<1\right\}$ and one can show that $x_{F}=\phi^{-1}(0)=\inf \{x \in[0,+\infty), \phi(x)=0\}$. By convention $x_{F}=+\infty$ if the generator is strict, so that the (ii) case can only occur with non-strict generator (which is consistent with the fact that bounded random variable $R$ corresponds to the existence of a non degenerated zero set, see further discussion).

One can first remark that, since $\phi$ is decreasing, the extremal behaviour of $R$ (resp. of $1 / R$ ) corresponds to the lower tail dependence (resp. upper tail dependence) of $\mathbf{U}=\left(\phi\left(R S_{1}\right), \ldots, \phi\left(R S_{d}\right)\right)$. One discusses here the link with previously obtained results, for $r>0$, and consequences on lower tail dependence coefficient.

(i) Fréchet case: For $r \in(0,+\infty), F_{R} \in \operatorname{MDA}\left(\Phi_{1 / r}\right)$ if and only if $\psi \in \mathcal{R} \mathcal{V}_{-r}(0)$, by Lemma $\mathrm{B}$. Then, the coefficient $r>0$ is both leading to lower tail dependence, (see Theorem 2.1) and to a Fréchet domain of attraction for $R$. In this case the multivariate lower tail coefficient with dimensions $h$ and $d$ is given by

$$
\lambda_{L}^{(h, d-h)}=d^{-1 / r}(d-h)^{1 / r} .
$$

Remark that equation before generalizes Corollary $2(i)$ in Larsson and Nešlehová (2011) and in Section 3 of Charpentier and Segers (2009), stated for a pair of variables arbitrarily chosen in the $d$-dimensional vector.

(ii) Weibull case: $F_{R} \in \operatorname{MDA}\left(\Psi_{\alpha}\right)$ if and only if $\phi \in \mathcal{R} \mathcal{V}_{-\alpha-d+1}\left(x_{F}\right)$. This situation occurs for non-strict generators where $x_{F}<\infty$. Indeed, a Weibull domain of attraction corresponds to a bounded random variable $R$, and thus to situations where the zero set $\left\{\left(u_{1}, \ldots, u_{d}\right): C\left(\left(u_{1}, \ldots, u_{d}\right)=0\right)\right\}$ is not restricted to the lines $\{0\} \times[0,1]$ and $[0,1] \times\{0\}$ (see Nelsen (1999), Section 4.3 and Exercise 4.5). One cannot necessarily define lower-tail dependence coefficients in this case, as $\mathbb{P}\left[U_{1} \leq u, \ldots, U_{d-h} \leq u\right]$ can be zero for any $u$ below a given threshold $u_{0}>0$. In other sections of the present paper, this case is excluded since the chosen generator is assumed to be strict.

(iii) Gumbel case: $F_{R} \in \operatorname{MDA}(\Lambda)$ if and only if $\phi \in \Delta$. As stated in Proposition 3.10.3 in Bingham et al. (1989), $\phi \in \Delta$ implies that $\phi$ is rapidly varying at infinity, $\phi \in \mathcal{R} \mathcal{V}{ }_{-\infty}(\infty)$, and thus that $\psi \in \mathcal{R} \mathcal{V}_{0}(0)$. This corresponds to the case where one have lower tail independence. In this case the multivariate lower tail coefficient with dimensions $h$ and $d$ is given by

$$
\lambda_{L}^{(h, d-h)}=0
$$

(see the second order behavior in the case $r=0$ provided in Theorem 2.2.

Analogously to the previous discussion, we can easily provide the link between the domain of attraction of $1 / R$ and the upper tail dependence coefficient.

In order to study the tail behaviour of transformed Archimedean copulas, in Lemma 5.1 (resp. Lemma 5.2) we characterize the extremal behavior of the transformed radial part $\widetilde{R}$ (resp. inverse of the transformed radial part $1 / \widetilde{R}$ ) as an application of Theorem 1 in Larsson and Nešlehová (2011) (resp. Theorem 2 in Larsson and Nešlehová (2011)). We restricted ourselves in Assumption 1.1 to strict generators, so that in Lemma 5.1, the Weibull domain of attraction is excluded for the considered transformed copula. 
Lemma 5.1 (Maximum domain of attraction for $\widetilde{R}$ ) Let $\phi_{0}$ be a strict initial generator as in Assumption 1.1 and $T$ be an admissible transformation as in Definition [3.1. Assume that $\psi_{0} \in \mathcal{R} \mathcal{V}_{-r_{0}}(0), r_{0} \in[0,+\infty]$ as in Definition 2.1 i) and that $T \in \mathcal{R} \mathcal{V}_{\widetilde{a}}(0)$, with $\widetilde{a} \in(0,+\infty)$. Let $\widetilde{R}$ be the radial part associated to the transformed Archimedean copula $\widetilde{C}_{T, C_{0}}$ in (6) and $F_{\widetilde{R}}$ be its distribution function. Then,

- $\widetilde{r}=\frac{r_{0}}{\widetilde{a}}>0$, if and only if $F_{\widetilde{R}} \in \operatorname{MDA}\left(\Phi_{1 / \widetilde{r}}\right)$ (Fréchet case, lower tail dependence).

- $\widetilde{r}=\frac{r_{0}}{\widetilde{a}}=0$ and $\widetilde{\phi} \in \Delta$ if and only if $F_{R} \in \operatorname{MDA}(\Lambda)$ (Gumbel case, lower tail independence).

Remark that, using Proposition 3.10.3 in Bingham et al. $(1989), \widetilde{\phi} \in \Delta$ implies that $\widetilde{\psi} \in \mathcal{R} \mathcal{V}_{0}(0)$, i.e., $\widetilde{r}=\frac{r_{0}}{\widetilde{a}}=0$ (see Theorem 3.4).

Lemma 5.2 (Maximum domain of attraction for $1 / \widetilde{R}$ ) Let $\phi_{0}$ be a strict initial generator as in Assumption 1.1 and $T$ be an admissible transformation as in Definition 3.1. Assume that $\psi_{0} \in \mathcal{R} \mathcal{V}_{\rho_{0}}(1), \rho_{0} \in[1,+\infty]$ as in Definition 2.1 ii), and that $T \in \mathcal{R} \mathcal{V}_{\widetilde{\alpha}}(1)$, with $\widetilde{\alpha} \in\left(0, \rho_{0}\right]$. Let $\widetilde{R}$ be the radial part associated to the transformed Archimedean copula $\widetilde{C}_{T, C_{0}}$ in $(6)$, and $F_{1 / \widetilde{R}}$ be the distribution function of $1 / \widetilde{R}$. Then,

- $\rho_{0}>\widetilde{\alpha}$ if and only if $F_{1 / \widetilde{R}} \in \operatorname{MDA}\left(\Phi_{\widetilde{\alpha} / \rho_{0}}\right)$ (Fréchet case, upper tail dependence).

- if $F_{1 / \widetilde{R}} \in \operatorname{MDA}\left(\Phi_{\widetilde{\xi}}\right)$ with $\widetilde{\xi} \geq 1$ or if $F_{1 / \widetilde{R}} \in \operatorname{MDA}(\Lambda)$ or if $F_{1 / \widetilde{R}} \in \operatorname{MDA}\left(\Psi_{\widetilde{\xi}}\right)$ with $\widetilde{\xi}>0$ then $\widetilde{\psi} \in \mathcal{R} \mathcal{V}_{\widetilde{\rho}}(1)$ with $\widetilde{\rho}=1$ i.e., $\rho_{0}=\widetilde{\alpha}$ (upper tail independence).

\subsection{Transformed geometrically ergodic Markov chain}

Theorem 3.1 can be an important tool to obtain the ergodic property of the stationary Markov chain $\left\{U_{t}: t \in \mathbb{Z}\right\}$ with transformed joint distribution $\widetilde{C}$ of $\left(U_{0}, U_{1}\right)$. For sake of clarity, in the following we firstly recall the definition of geometric ergodicity (see Definition 5.1) and the geometric ergodic theorem for Archimedean copulas (see Theorem 5.1).

Definition 5.1 (Definition 3.1. in Beare (2010)) The stationary Markov chain $\left\{U_{t}: t \in \mathbb{Z}\right\}$ is said to be geometrically ergodic if, for a.e. $u \in(0,1)$, there exists a real number $l>1$ such that

$$
\sum_{j=1}^{\infty} l^{j} \sup _{B \in \mathcal{B}}\left|\mathbb{P}\left[U_{j} \in B \mid U_{0}=u\right]-\mathbb{P}\left[U_{j} \in B\right]\right|<\infty,
$$

where $\mathcal{B}$ denote the $\sigma$-field of Borel subsets of $(0,1)$.

For a stationary real valued Markov chain, geometric ergodicity is equivalent to exponentially fast $\beta$-mixing.

Theorem 5.1 (Theorem 3.1. in Beare (2010)) Suppose $\left\{U_{t}: t \in \mathbb{Z}\right\}$ is a stationary Markov chain whose invariant distribution is uniform on $(0,1)$. Let $C$ denote the joint distribution function of $\left(U_{0}, U_{1}\right)$. Assume that the copula $C$ is strictly Archimedean, with an inverse generator $\psi$ satisfying the following conditions:

i) $\psi \in \mathcal{R V}_{-r}(0)$ with $r \in[0,+\infty)$, and $\psi \in \mathcal{R} \mathcal{V}_{\rho}(1)$, with $\rho \in[1,+\infty)$;

ii) $\psi$ is twice continuously differentiable on $(0,1)$;

iii) $\psi^{\prime \prime}$ is monotone in a right-neighborhood of zero and in a left-neighborhood of one;

iv) $\psi^{\prime \prime}$ is strictly positive on $(0,1)$;

v) If $r=0$, then

a) $-\psi^{\prime} \in \mathcal{R} \mathcal{V}_{-1}(0)$, and

b) $u \psi^{\prime}(u)$ is bounded away from zero for $u$ in a right-neighborhood of zero.

vi) If $\rho=1$, then $\psi^{\prime}$ and $\psi^{\prime \prime}$ are bounded away from zero in a left-neighborhood of one.

Then $\left\{U_{t}: t \in \mathbb{Z}\right\}$ is geometrically ergodic. 
Remark that Clayton, Ali-Mikhail-Haq, Gumbel, Frank and Joe copulas among others, with suitable parameter ranges, satisfy assumptions of Theorem 5.1 (see Examples 3.1-3.11 in Beare (2010)). Conversely, generator (20) in Table 4.1 of Nelsen (1999) does not satisfy Assumptions of Theorem 5.1 Indeed in this case $\log \psi \in \mathcal{R} \mathcal{V}_{-\theta}(0)$, and $\psi$ is said to be rapidly varying at zero, $\psi \in \mathcal{R} \mathcal{V}_{\infty}(0)$ (see for instance Section 2.4 in Bingham et al. (1989) for a formal definition of rapid variation). In particular Theorem 5.1 is an application of the Geometric Ergodic Theorem, discussed in detail in the text of Meyn and Tweedie (2009). The proof involves verifying that the one-step dependence characterized by copula $C$ satisfies a Foster-Lyapunov drift condition. Chen et al. (2009) used precisely this approach to prove geometric ergodicity for the Clayton and Gumbel families. Then Theorem 5.1 provides geometric ergodicity for a large class of Archimedean copulas.

Using Theorem 3.1 we obtain the regular varying properties of the transformed inverse generator $\widetilde{\psi}$. In Corollary 5.1 below, we underline that these properties can be used to get geometrically ergodic stationary Markov chain with transformed joint distribution $\widetilde{C}$ of $\left(U_{0}, U_{1}\right)$. Remark that obviously differentiable and monotonic conditions of Theorem 5.1 have to be also verified.

Corollary 5.1 Let $C_{0}$ be the initial Archimedean copula with inverse associated generator $\psi_{0}$, satisfying assumptions of Theorem 5.1. Under assumptions of Theorem 3.1 also the transformed inverse generator $\widetilde{\psi}=\psi_{0} \circ T^{-1}$ satisfies assumption i) in Theorem 5.1. If $\widetilde{\psi}$ satisfies also the remaining assumptions of Theorem 5.1, then $\left\{U_{t}: t \in \mathbb{Z}\right\}$ with transformed joint distribution $\widetilde{C}$ of $\left(U_{0}, U_{1}\right)$ is also geometrically ergodic.

The proof is postponed to Appendix B.

\section{Conclusion}

In this paper we deal with the study of the tails of certain transformed Archimedean copulas. We consider the class of transformations previously proposed for instance in Durante et al. (2010), Di Bernardino and Rullière (2013a), Di Bernardino and Rullière (2013b). In particular the relationship between the regular variation of the transformation $T$ and of the transformed tails is investigated. These results extend some bivariate results of Durante et al. (2010) using the definition of upper and lower multivariate tail dependence coefficients previously proposed by De Luca and Rivieccio (2012). The first part of the paper exploits previous works of Charpentier and Segers (2007), and extends some bivariate results of Juri and Wüthrich (2003). Indeed in this part, we calculate tail dependence coefficients in this Archimedean setting when the generator of considered copula exhibits some regular variation properties. In the second part of the paper we obtain some new results by analysing the impact in the upper and lower multivariate tail dependence coefficients of a large class of transformations of dependence structures. Furthermore we analyse the behavior in a particular parametric class of transformations proposed by Di Bernardino and Rullière (2013a) under specific conditions involving regularly varying hazard rates of components of these transformations. Finally we investigate the importance of using transformed Archimedean copulas. Indeed they permit to construct Archimedean generator exhibiting any chosen couple of lower and upper multivariate transformed tail dependence coefficients. Some perspectives are the derivation of a complete estimation procedure that uses these generators with given tail coefficients, and further developments in a dynamical setting.

\section{A Illustration for some usual copulas}

A complete survey of Archimedean regularly varying generators would be out of the scope of the present paper. However a large list of common parametric families of Archimedean generators and associated regular varying properties is given in Table 1 in Charpentier and Segers (2009). In the following we consider two lower and upper regularly varying generator (Gumbel and Clayton copulas) and we illustrate their properties using Lemma B.

Gumbel (and independent) generator Let us consider the Gumbel inverse generator $\psi(t)=(-\ln t)^{\theta}$ and $\psi^{-1}(x)=\exp \left(-x^{1 / \theta}\right)$, with $\theta \geq 1$. Calculating derivatives, one gets $\psi^{\prime}(t)=-\frac{\theta}{t}(-\ln t)^{\theta-1}$. Using Lemma $\mathrm{B}$, one easily remarks that the Gumbel generator is such that:

$$
\psi^{\operatorname{Gumbel}(\theta)} \in \mathcal{R} \mathcal{V}_{0}(0), \quad \text { and } \quad \psi^{\operatorname{Gumbel}(\theta)} \in \mathcal{R} \mathcal{V}_{\theta}(1), \text { for } \theta \in[1,+\infty)
$$

Remark that the comonotonic copula is not an Archimedean one, then the case $\theta=+\infty$ is excluded in Equation 20 . By the lower and upper regularly variation of $\psi^{\operatorname{Gumbel}(\theta)}=(-\ln t)^{\theta}$, for $\theta \in[1,+\infty)$, and Theorem 2.1 we get for 
$h \geq 1$ and $d-h \geq 1$,

$$
\lambda_{L}^{(h, d-h)}=0 \quad \text { and } \quad \lambda_{U}^{(h, d-h)}=\frac{\sum_{i=1}^{d}(-1)^{i} C_{d}^{i} \cdot i^{1 / \theta}}{\sum_{i=1}^{d-h}(-1)^{i} C_{d-h}^{i} \cdot i^{1 / \theta}} .
$$

Remark that the Gumbel copula for $\theta=1$ is the independent one. Then the regular variation index at one and at zero for the inverse independence generator $\psi(t)=-\ln (t)$ is given by:

$$
\psi^{\text {Indep }} \in \mathcal{R} \mathcal{V}_{0}(0) \quad \text { and } \quad \psi^{\text {Indep }} \in \mathcal{R} \mathcal{V}_{1}(1)
$$

Obviously, in the independent case, for $h \geq 1$ and $d-h \geq 1$,

$$
\lambda_{L}^{(h, d-h)}=0 \quad \text { and } \quad \lambda_{U}^{(h, d-h)}=0 .
$$

An illustration of the generator $\phi^{\operatorname{Gumbel}(\theta)}$ and its inverse $\psi^{\operatorname{Gumbel}(\theta)}$ is presented in Figure 3 both for the lower and upper regularly variation, for different values of parameter $\theta$. Some indications lower and upper are given in order to illustrate the fact that right derivatives of $\psi^{\operatorname{Gumbel}(\theta)}$ at zero are linked to lower-tail coefficients, whereas left derivatives at one are linked to upper-tail coefficients. In every case, upper-tail behaviour of the copula is linked to the generator behaviour around the attachment point $(t, \psi(t))=(1,0)$.
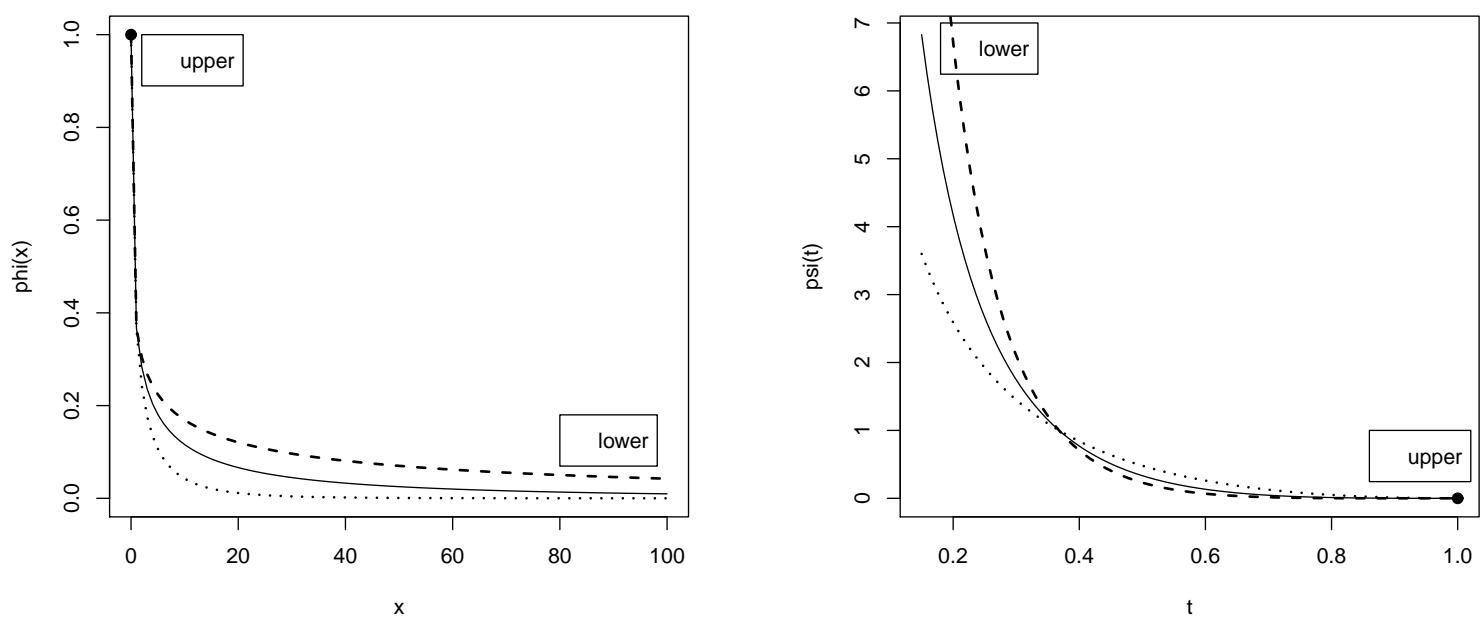

Figure 3: Generators $\phi^{\operatorname{Gumbel}(\theta)}=\exp \left(-x^{1 / \theta}\right)$ (left) and its inverse $\psi^{\operatorname{Gumbel}(\theta)}=(-\ln t)^{\theta}$ (right) for a Gumbel copula with parameters $\theta=4$ (dashed lines), $\theta=3$ (full lines) and $\theta=2$ (dotted lines). As given in Equation 200, we indicate the upper and lower parts of the generator associated to the regular varying property of Lemma $B$.

In the Gumbel case, one easily shows that $\mu_{\phi}=\phi^{\prime} / \phi \in \mathcal{R} \mathcal{V}_{k-1}(\infty)$ with $k=\frac{1}{\theta}$, so that in this case

$$
\lambda_{L}^{(h, d-h)}(u)=\frac{\delta^{(d)}(u)}{\delta^{(d-h)}(u)} \in \mathcal{R} \mathcal{V}_{d^{\frac{1}{\theta}}-(d-h)^{\frac{1}{\theta}}}(0) .
$$

In the independence case, when $\theta=1$, then

$$
\lambda_{L}^{(h, d-h)}(u)=\frac{\delta^{(d)}(u)}{\delta^{(d-h)}(u)} \in \mathcal{R} \mathcal{V}_{h}(0) .
$$

Clayton generator Let us consider the Clayton inverse generator $\psi(t)=\frac{1}{\theta}\left(t^{-\theta}-1\right)$ and $\psi^{-1}(x)=(1+\theta x)^{-1 / \theta}$, with $\theta \in(0,+\infty)$. Then the generator of Clayton's copula is a regular varying generator at zero with index $-\theta$ and at one with index 1, i.e.,

$$
\psi^{\text {Clayton }(\theta)} \in \mathcal{R} \mathcal{V}_{-\theta}(0), \quad \text { and } \quad \psi^{\text {Clayton }(\theta)} \in \mathcal{R} \mathcal{V}_{1}(1), \forall \theta \in(0,+\infty)
$$

Similarly, by application of Theorem 2.1, we obtain for $h \geq 1$ and $d-h \geq 1$,

$$
\lambda_{L}^{(h, d-h)}=d^{1 / \theta}(d-h)^{-1 / \theta} \quad \text { and } \quad \lambda_{U}^{(h, d-h)}=0 .
$$


An illustration of the generator $\phi^{\text {Clayton( }(\theta)}$ and its inverse $\psi^{\text {Clayton( } \theta)}$ is presented in Figure 4 both for the lower and upper regularly variation, for different values of parameter $\theta$. Even in this case some indications lower and upper are given.
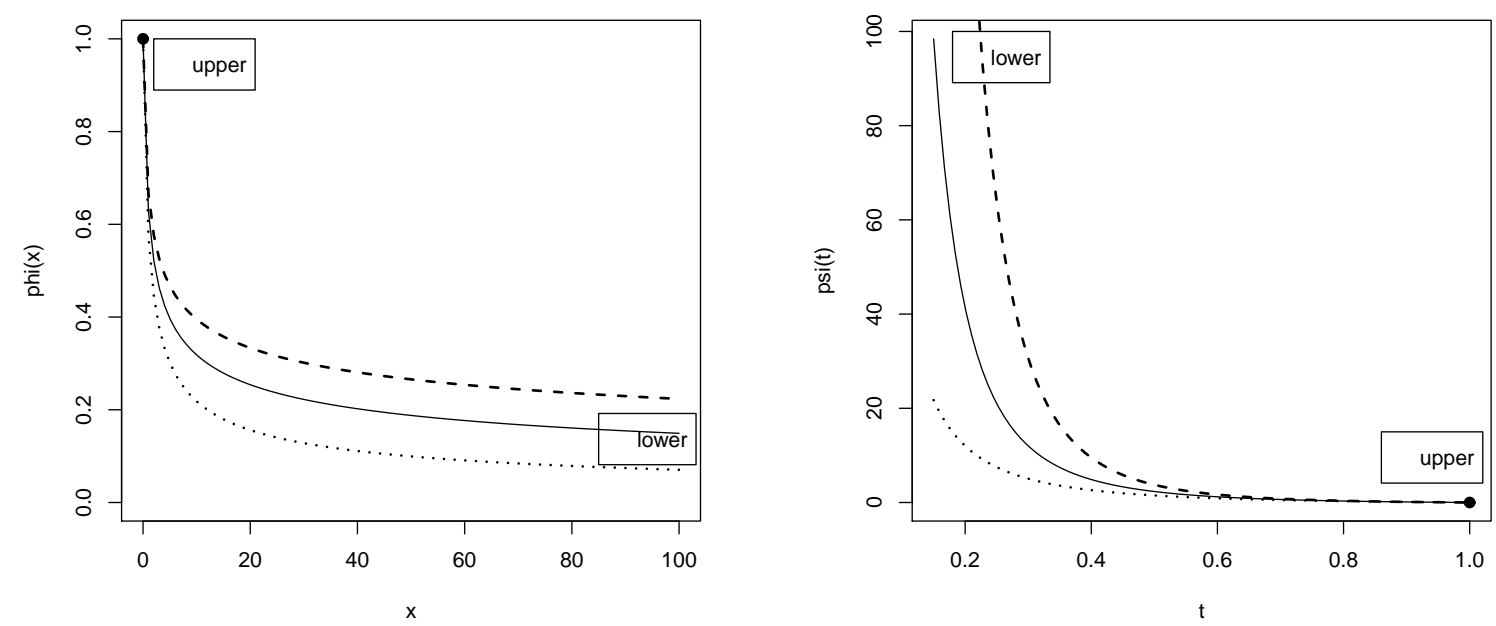

Figure 4: Generators $\phi^{\operatorname{Clayton}(\theta)}(x)=(1+\theta x)^{-1 / \theta}$ (left) and its inverse $\psi^{\text {Clayton( }(\theta)}(t)=\frac{1}{\theta}\left(t^{-\theta}-1\right)$ (right) for a Clayton copula with parameters $\theta=4$ (dashed lines), $\theta=3$ (full lines) and $\theta=2$ (dotted lines). As given in Equation 21, we indicate the upper and lower parts of the generator associated to the regular varying property of Lemma B,

In the Clayton case, one easily shows that $m_{\phi}=-\phi^{\prime} /(1-\phi) \in \mathcal{R} \mathcal{V}_{-\kappa-1}(0)$ with $\kappa=0$. Furthermore from Theorem 2.3 (case $\rho=1$ ) one can check that $(-D)^{d} \phi(0)$ is finite and not zero, where $D$ is the derivative operator, then we get

$$
\lambda_{U}^{(h, d-h)}(u) \in \mathcal{R} \mathcal{V}_{h}(1)
$$

The asymptotic regular varying behaviour of a generator is difficult to interpret graphically on a plot of $\psi$ or $\phi$. We first recall that for any Archimedean copula, there exists a whole family of equivalent generators that are different but leading to the same copula, see Di Bernardino and Rullière (2013b). Furthermore, the regular variation of $\psi$ at 0 is difficult to compare to the regular variation at 1 (see Figures 34 ). This is first due to the fact that the scale is not the same as $\psi$ tends to $+\infty$ at 0 whereas it tends to 0 at 1 . One can of course re-scale these figures, by drawing for example $1-\exp (-\psi(t))$ as a function of $t$ so that the curve decreases from $(0,1)$ to $(1,0)$. But even after a re-scaling, differences between upper-tail and lower-tail behaviour would be hard to distinguish.

Finally, in Table 2 we gathered the considered indexes for Gumbel and Clayton generators.

\begin{tabular}{|c|c|c|c|c|}
\hline & Lower & Lower & Upper & Upper \\
& $r$ & $k$ & $\rho$ & $\kappa$ \\
\hline Gumbel, $\theta \in[1,+\infty)$ & 0 & $\frac{1}{\theta}$ & $\theta$ & 0 \\
\hline Clayton, $\theta \in(0,+\infty)$ & $\theta$ & 0 & 1 & 0 \\
\hline Independence & 0 & 1 & 1 & 0 \\
\hline
\end{tabular}

Table 2: Considered indexes for Gumbel and Clayton generators. On the lower side, $r \in[0,+\infty]$ and $k \in[0,+\infty)$ are such that $\psi \in \mathcal{R} \mathcal{V}_{-r}(0)$ and $\mu_{\phi}=-\phi^{\prime} / \phi \in \mathcal{R} \mathcal{V}_{k-1}(\infty)$. On the upper side, $\rho \in[1,+\infty]$ and $\kappa \in[0,+\infty)$ are such that $\psi \in \mathcal{R} \mathcal{V}_{\rho}(1)$ and $m_{\phi}=-\phi^{\prime} /(1-\phi) \in \mathcal{R} \mathcal{V}_{-\kappa-1}(0)$.

\section{B Proofs}

Proof of Lemma 2.1 On the lower side, this result can be seen as a direct consequence of Theorem 3.3. in Charpentier and Segers (2009). As $\delta^{(i)}(\cdot)$ is a decreasing function of $i$, if $\delta^{(i)} \in \mathcal{R} \mathcal{V}_{z_{i}}(0)$ then $z_{i}=i^{k}$ must be an 
increasing function of $i$, with $k \geq 0$, which correspond to the index domain in Charpentier and Segers $(2009)$. On the upper side, recall $M(x)=1-x$ and $I(x)=1 / x$, and denote by $K=M \circ \delta^{(i)} \circ M \circ I$. Setting $y=\phi^{-1} \circ M \circ I(x)$, one sees that $y \rightarrow 0^{+}$as $x \rightarrow+\infty$, and

$$
\lim _{x \rightarrow+\infty} \frac{x K^{\prime}(x)}{K(x)}=-i \cdot \lim _{y \rightarrow 0^{+}} \frac{m_{\phi}(i y)}{m_{\phi}(y)} .
$$

So that if $m_{\phi} \in \mathcal{R} \mathcal{V}_{-\kappa-1}(0), \lim _{x \rightarrow+\infty} \frac{x K^{\prime}(x)}{K(x)}=-i^{-\kappa}$, thus $K=M \circ \delta^{(i)} \circ M \circ I \in \mathcal{R} \mathcal{V}_{-i^{-\kappa}}(\infty)$, and $M \circ \delta^{(i)} \in \mathcal{R} \mathcal{V}_{i^{-\kappa}}(1)$ Hence the result. As $M \circ \delta^{(i)}(\cdot)$ is an increasing function of $i$, if $M \circ \delta^{(i)} \in \mathcal{R} \mathcal{V}_{\zeta_{i}}(1)$ then $\zeta_{i}=i^{-\kappa}$ must be a decreasing function of $i$, with $\kappa \geq 0$.

Proof of Theorem $[\mathbf{2 . 1}$ From Definition 1.1 and Lemma B iii), we get

$$
\lambda_{L}^{(h, d-h)}=\lim _{u \rightarrow 0^{+}} \frac{\psi^{-1}(d \psi(u))}{\psi^{-1}(\psi(u))} \frac{\psi^{-1}(\psi(u))}{\psi^{-1}((d-h) \psi(u))}=d^{-1 / r}(d-h)^{1 / r},
$$

with proper interpretations for $r$ equal to zero or infinity. Hence the first result. Remark that, for $d \geq 1$, $\sum_{i=0}^{d}(-1)^{i} C_{d}^{i}=(1-1)^{d}=0$. Then $r_{d}(u)=\sum_{i=0}^{d}(-1)^{i} C_{d}^{i} \psi^{-1}(i \psi(u))=-\sum_{i=0}^{d}(-1)^{i} C_{d}^{i}\left(1-\psi^{-1}(i \psi(u))\right)$. So, from Definition 1.1 (see also De Luca and Rivieccio (2012)), one have

$$
\lambda_{U}^{(h, d-h)}=\lim _{x \rightarrow \infty} \frac{r_{d}(M \circ I(x))}{r_{d-h}(M \circ I(x))} .
$$

Finally,

$$
\lambda_{U}^{(h, d-h)}=\lim _{x \rightarrow \infty} \frac{\sum_{i=0}^{d}(-1)^{i} C_{d}^{i} \cdot\left(1-\psi^{-1}(i \cdot \psi \circ M \circ I(u))\right) \cdot x}{\sum_{i=0}^{d-h}(-1)^{i} C_{d-h}^{i} \cdot\left(1-\psi^{-1}(i \cdot \psi \circ M \circ I(u))\right) \cdot x} .
$$

When the denominator is not zero, results comes from application of Lemma B iii). Hence the result.

Proof of Theorem 2.2 Notice that $\mu_{\phi}=-\frac{\phi^{\prime}}{\phi} \in \mathcal{R} \mathcal{V}_{k-1}(\infty)$ implies that $-\frac{\phi}{\phi^{\prime}} \in \mathcal{R} \mathcal{V}_{-k+1}(\infty)$. Recall that the diagonal section in the dimension $i$ is $\delta^{(i)}(u)=\psi^{-1}(i \psi(u))$, then from Lemma 2.1 one gets for $i \in \mathbb{N} \backslash\{0\}$ that

$$
\delta^{(i)} \in \mathcal{R} \mathcal{V}_{z_{i}}(0) \text { with } z_{i}=i^{-k} \text {. }
$$

It can then be shown that if there exist $z_{d}, z_{d-h} \geq 0$ such that $\delta^{(d)} \in \mathcal{R} \mathcal{V}_{z_{d}}(0)$ and $\delta^{(d-h)} \in \mathcal{R} \mathcal{V}_{z_{d-h}}(0)$, then

$$
\lambda_{L}^{(h, d-h)}(u)=\frac{\delta^{(d)}(u)}{\delta^{(d-h)}(u)} \in \mathcal{R} \mathcal{V}_{z_{d}-z_{d-h}}(0)
$$

This last result comes down easily by calculating $\lambda_{L}^{(h, d-h)}(s u) / \lambda_{L}^{(h, d-h)}(u)$ for a real $s>0$.

Proof of Theorem 2.3 Under required assumptions, from Theorem 4.3 in Charpentier and Segers (2009), we obtain

$$
\lim _{u \rightarrow 0} u^{-d} \mathbb{P}\left[U_{1} \geq 1-u s, \ldots, U_{d} \geq 1-u s\right]=\left|\psi^{\prime}(1)\right|^{d}(-D)^{d} \phi(0) s^{d} .
$$

Then $\lim _{u \rightarrow 0} u^{-d} r_{d}(1-u s)=\left|\psi^{\prime}(1)\right|^{d}(-D)^{d} \phi(0) s^{d}$ and $\lim _{u \rightarrow 0} u^{-(d-h)} r_{d-h}(1-u s)=\left|\psi^{\prime}(1)\right|^{d-h}(-D)^{d-h} \phi(0) s^{d-h}$. So

$$
\lim _{u \rightarrow 0} \frac{u^{-d} r_{d}(1-u s)}{u^{-(d-h)} r_{d-h}(1-u s)}=\frac{\left|\psi^{\prime}(1)\right|^{d}(-D)^{d} \phi(0) s^{d}}{\left.\left|\psi^{\prime}(1)\right|\right|^{d-h}(-D)^{d-h} \phi(0) s^{d-h}}=\alpha_{d, d-h} s^{h} .
$$

Finally, since $\lambda_{U}^{(h, d-h)}(u)=\frac{r_{d}(u)}{r_{d-h}(u)}$, we get $\lim _{u \rightarrow 0} u^{-h} \lambda_{U}^{(h, d-h)}(1-u s)=\alpha_{d, d-h} s^{h}$. Since by assumptions, $\alpha_{d, d-h}$ is finite and not zero, then

$$
\lim _{u \rightarrow 0} \frac{\lambda_{U}^{(h, d-h)}(1-u s)}{\lambda_{U}^{(h, d-h)}(1-u)}=s^{h} .
$$

Hence the first result. For the second result, from Corollary 4.7 in Charpentier and Segers (2009), for $d-h \geq 2$,

$$
\lim _{u \rightarrow 0} \lambda_{U}^{(h, d-h)}(1-u s)=\frac{\mathfrak{r}_{d}(s, \ldots, s)}{\mathfrak{r}_{d-h}(s, \ldots, s)},
$$

with $\mathfrak{r}_{d}(s, \ldots, s)=s \sum_{k=1}^{d} C_{d}^{k}(-1)^{k} k \ln (k)$. Then finally, 


$$
\lim _{u \rightarrow 0} \lambda_{U}^{(h, d-h)}(1-u s)=\frac{\sum_{k=1}^{d} C_{d}^{k}(-1)^{k} k \ln k}{\sum_{k=1}^{d-h} C_{d-h}^{k}(-1)^{k} k \ln (k)}
$$

and

$$
\lim _{u \rightarrow 0} \frac{\lambda_{U}^{(h, d-h)}(1-u s)}{\lambda_{U}^{(h, d-h)}(1-u)}=1 .
$$

So $\lambda_{U}^{(h, d-h)}(u) \circ M \in \mathcal{R} \mathcal{V}_{0}(0)$ and equivalently $\lambda_{U}^{(h, d-h)}(u) \in \mathcal{R} \mathcal{V}_{0}(1)$. Hence the second result.

Proof of Theorem 3.1 To prove (7), one defines

$$
K(x)=\widetilde{\psi} \circ I(x)=\psi_{0} \circ T^{-1} \circ I(x),
$$

then

$$
\lim _{x \rightarrow \infty} \frac{x K^{\prime}(x)}{K(x)}=\lim _{x \rightarrow \infty}-\frac{\psi_{0}^{\prime} \circ T^{-1} \circ I(x) \cdot T^{-1} \circ I(x)}{\psi_{0} \circ T^{-1} \circ I(x)} \cdot \frac{\left[T^{-1}\right]^{\prime} \circ I(x)}{x \cdot T^{-1} \circ I(x)} .
$$

Setting $z=T^{-1} \circ I(x), z$ tends to $0^{+}$as $x$ tends to $\infty$, using Definition 2.1 i) and from Lemma B.

$$
\lim _{z \rightarrow 0^{+}} \frac{z \cdot \psi_{0}^{\prime}(z)}{\psi_{0}(z)}=-r_{0}
$$

Finally,

$$
\lim _{x \rightarrow \infty} \frac{x K^{\prime}(x)}{K(x)}=\frac{r_{0}}{\widetilde{a}}
$$

and $\widetilde{\psi} \in \mathcal{R} \mathcal{V}_{-\widetilde{r}}(0)$, with $\widetilde{r}=\frac{r_{0}}{\widetilde{a}} \geq 0$. Hence the result in (7). To prove the result in (8), one defines

$$
H(x)=\widetilde{\psi} \circ M \circ I(x)=\psi_{0} \circ T^{-1} \circ M \circ I(x) .
$$

Then

$$
\lim _{x \rightarrow \infty} \frac{x H^{\prime}(x)}{H(x)}=\frac{\psi_{0}^{\prime} \circ T^{-1} \circ M \circ I(x) \cdot\left(1-T^{-1} \circ M \circ I(x)\right)}{\psi_{0} \circ T^{-1} \circ M \circ I(x)} \cdot \frac{\left[T^{-1}\right]^{\prime} \circ M \circ I(x)}{x \cdot\left(1-T^{-1} \circ M \circ I(x)\right)} .
$$

Now set $y=T^{-1} \circ M \circ I(x), y$ tends to $1^{-}$as $x$ tends to $\infty$. We get, using Definition 2.1 ii) and from Lemma B

$$
\lim _{x \rightarrow \infty} \frac{x H^{\prime}(x)}{H(x)}=-\frac{\rho_{0}}{\widetilde{\alpha}}
$$

Finally $\tilde{\psi} \circ M \circ I \in \mathcal{R} \mathcal{V}_{-\frac{\rho_{0}}{\alpha}}(\infty)$, so that $\tilde{\psi} \in \mathcal{R} \mathcal{V}_{\frac{\rho_{0}}{\alpha}}(1)$. Hence the result in (8). Furthermore, since by assumption $\widetilde{\alpha} \in\left(0, \rho_{0}\right]$ then $\widetilde{\rho} \in[1,+\infty]$, i.e., $\widetilde{\psi}$ is a valid regularly varying generator (see Lemma A).

Proof of Theorem 3.3 On the lower side, one easily shows that the hazard rate for the transformed generator $\widetilde{\phi}=T \circ \phi_{0}$ is

$$
\mu_{\widetilde{\phi}}(x)=\frac{\phi_{0}(x) \cdot T^{\prime} \circ \phi_{0}(x)}{T \circ \phi_{0}(x)} \mu_{\phi_{0}}(x) .
$$

Assume that $T \in \mathcal{R} \mathcal{V}_{\widetilde{a}}(0)$ with $\widetilde{a} \in(0,+\infty)$, then $u T^{\prime}(u) / T(u) \rightarrow \widetilde{a}$ as $u$ tends to 0 . As a consequence, since $\widetilde{a} \neq 0$ and $\mu_{\phi_{0}} \in \mathcal{R} \mathcal{V}_{k_{0}-1}(\infty)$ with $k_{0} \geq 0$

$$
\lim _{x \rightarrow+\infty} \frac{\mu_{\tilde{\phi}}(s x)}{\mu_{\tilde{\phi}}(x)}=\lim _{x \rightarrow+\infty} \frac{\mu_{\phi_{0}}(s x)}{\mu_{\phi_{0}}(x)}=s^{\left(k_{0}-1\right)} .
$$

On the upper side, one easily shows that the rate for the transformed generator $\widetilde{\phi}=T \circ \phi_{0}$ is

$$
m_{\widetilde{\phi}}(x)=\frac{\left(1-\phi_{0}(x)\right) \cdot T^{\prime} \circ \phi_{0}(x)}{1-T \circ \phi_{0}(x)} m_{\phi_{0}}(x) .
$$

Assume that $M \circ T \in \mathcal{R} \mathcal{V}_{\widetilde{\alpha}}(1)$ with $\widetilde{\alpha} \in(0,+\infty)$, then $(1-z) T^{\prime}(z) / T(z) \rightarrow \widetilde{\alpha}$ as $z$ tends to 1 . As a consequence, since $\widetilde{\alpha} \neq 0$ and $m_{\phi_{0}} \in \mathcal{R} \mathcal{V}_{-\kappa_{0}-1}(0)$ with $\kappa_{0} \geq 0$,

$$
\lim _{x \rightarrow 0} \frac{m_{\widetilde{\phi}}(s x)}{m_{\widetilde{\phi}}(x)}=\lim _{x \rightarrow 0} \frac{m_{\phi_{0}}(s x)}{m_{\phi_{0}}(x)}=s^{\left(-\kappa_{0}-1\right)} .
$$


Then we obtain the result.

Proof of Theorem 3.6 For the first part of the proof, $T_{f, G} \in \mathcal{R} \mathcal{V}_{\widetilde{a}}(0)$ if $T_{f, G} \circ I \in \mathcal{R} \mathcal{V}_{-\widetilde{a}}(\infty)$. One thus considers the function $H=T_{f, G} \circ I=G \circ f \circ G^{-1} \circ I$, and the limit $\lim _{x \rightarrow \infty} \frac{x H^{\prime}(x)}{H(x)}$ which tends to the regular variation index $-\widetilde{a}$ of $T_{f, G}$ if this transformation is regularly varying at 0 . We obtain

$$
\frac{x H^{\prime}(x)}{H(x)}=-\frac{\left(G^{\prime} \circ f \circ G^{-1} \circ I\right)}{\left(G \circ f \circ G^{-1} \circ I\right)} \cdot\left(f^{\prime} \circ G^{-1} \circ I\right) \cdot \frac{G \circ G^{-1} \circ I}{G^{\prime} \circ G^{-1} \circ I}(x)
$$

Setting $y=G^{-1} \circ I(x), y$ tends to $-\infty$ as $x$ tends to $+\infty$ and under Assumption 3.1 .

$$
\lim _{x \rightarrow+\infty} \frac{x H^{\prime}(x)}{H(x)}=-\lim _{y \rightarrow-\infty} \frac{m_{G}(f(y))}{m_{G}(y)} \cdot f^{\prime}(y)=-\lim _{y \rightarrow-\infty}\left(\frac{f(y)}{y}\right)^{g-1} \cdot f^{\prime}(y)=-a^{g} .
$$

For the second part of the proof, $M \circ T_{f, G} \in \mathcal{R} \mathcal{V}_{\widetilde{\alpha}}(1)$ is equivalent to $M \circ T_{f, G} \circ M \circ I \in \mathcal{R}_{-\widetilde{\alpha}}(\infty)$. One thus considers now the function $K=M \circ T_{f, G} \circ M \circ I=M \circ G \circ f \circ G^{-1} \circ M \circ I$, and the limit $\lim _{x \rightarrow \infty} \frac{x K^{\prime}(x)}{K(x)}$ which tends to the regular variation index $-\widetilde{\alpha}$ of $T_{f, G}$ if this transformation is regularly varying at 1 . We get

$$
\frac{x K^{\prime}(x)}{K(x)}=-\frac{\left(G^{\prime} \circ f \circ G^{-1} \circ M \circ I\right)}{\left(M \circ G \circ f \circ G^{-1} \circ M \circ I\right)} \cdot\left(f^{\prime} \circ G^{-1} \circ M \circ I\right) \cdot \frac{M \circ G \circ G^{-1} \circ M \circ I}{G^{\prime} \circ G^{-1} \circ M \circ I}(x) .
$$

Setting $y=G^{-1} \circ M \circ I(x), y$ tends to $+\infty$ as $x$ tends to $+\infty$ and under Assumption 3.2 ,

$$
\lim _{x \rightarrow+\infty} \frac{x K^{\prime}(x)}{K(x)}=-\lim _{y \rightarrow+\infty} \frac{\mu_{G}(f(y))}{\mu_{G}(y)} \cdot f^{\prime}(y)=-\lim _{y \rightarrow+\infty}\left(\frac{f(y)}{y}\right)^{\gamma-1} \cdot f^{\prime}(y)=-\alpha^{\gamma} .
$$

Hence the result.

Proof of Lemma 3.1 The first result comes by checking that $P_{g, \gamma}^{\prime} \in \mathcal{R} \mathcal{V}_{\gamma-1}(\infty)$ and $P_{g, \gamma}^{\prime} \in \mathcal{R} \mathcal{V}_{g-1}(-\infty)$, for $\gamma, g>0$. The second result comes directly by writing $\mu_{G}=\frac{G^{\prime}}{G}=\frac{F^{\prime} \circ P}{M \circ F \circ P} \cdot P^{\prime}=\mu_{F} \cdot P^{\prime}$ and $m_{G}=\frac{G^{\prime}}{G}=\frac{F^{\prime} \circ P}{F \circ P} \cdot P^{\prime}=$ $m_{F} \cdot P^{\prime}$.

Proof of Corollary 5.1 Since $\psi_{0}$ satisfies assumptions of Theorem 5.1, under assumptions of Theorem 3.1 , the transformed generator $\psi$ is such that $\widetilde{\psi} \in \mathcal{R} \mathcal{V}_{\widetilde{\rho}}(1)$ with $\widetilde{\rho}=\frac{\rho_{0}}{\widetilde{\alpha}}, \rho_{0} \in[1,+\infty)$ and $\widetilde{\alpha} \in\left(0, \rho_{0}\right]$ (see $(8)$ ). Then $\widetilde{\rho} \in[1,+\infty)$. Furthermore we get $\widetilde{\psi} \in \mathcal{R} \mathcal{V}_{-\widetilde{r}}(0)$ with $\widetilde{r}=\frac{r_{0}}{\widetilde{a}}$ with $r_{0} \in[0,+\infty)$ and $\widetilde{a} \in(0,+\infty)$ (see $(7)$ ). Then $\widetilde{r} \in[0,+\infty)$. So, $\widetilde{\psi}$ satisfies assumption $i$ ) of Theorem 5.1 . If the obtained transformed generator $\widetilde{\psi}$ satisfies also the others assumptions of Theorem 5.1 we obtain the result.

\section{References}

Abdous, B., Fougères, A., and Ghoudi, K. (2005). Extreme behaviour for bivariate elliptical distributions. Canad. J. Statist., 33(3):317-334 .

Alsina, C., Schweizer, B., and Frank, M. J. (2006). Associative functions: triangular norms and copulas. World Scientific.

Asmussen, S. and Kortschak, D. (2013). Error rates and improved algorithms for rare event simulation with heavy weibull tails. Methodology and Computing in Applied Probability, pages 1-21.

Avérous, J. and Dortet-Bernadet, J.-L. (2004). Dependence for Archimedean copulas and aging properties of their generating functions. Sankhyā: The Indian Journal of Statistics, pages 607-620.

Beare, B. K. (2010). Archimedean Copulas and Temporal Dependence. University of California at San Diego, Economics Working Paper Series.

Bienvenüe, A. and Rullière, D. (2011). Iterative adjustment of survival functions by composed probability distortions. The Geneva Risk and Insurance Review, 37(2):156-179.

Bienvenüe, A. and Rullière, D. (2012). On hyperbolic iterated distortions for the adjustment of survival functions. In Perna, C. and Sibillo, M., editors, Mathematical and Statistical Methods for Actuarial Sciences and Finance, pages 35-42. Springer Milan.

Bingham, N. H., Goldie, C. M., and Teugels, J. L. (1989). Regular variation, volume 27. Cambridge university press.

Charpentier, A. and Segers, J. (2007). Lower tail dependence for Archimedean copulas: characterizations and pitfalls. Insurance Math. Econom., 40(3):525-532. 
Charpentier, A. and Segers, J. (2009). Tails of multivariate Archimedean copulas. Journal of Multivariate Analysis, $100(7): 1521$ - 1537.

Chen, X., Wu, W. B., and Yi, Y. (2009). Efficient estimation of copula-based semiparametric Markov models. The Annals of Statistics, $37: 4214-4253$.

Coles, S., Heffernan, J., and Tawn, J. (1999). Dependence measures for extreme value analyses. Extremes, 2(4):339-365.

de Haan, L. and Ferreira, A. (2006). Extreme Value Theory: An Introduction. Springer Series in Operations Research and Financial Engineering. Springer.

De Luca, G. and Rivieccio, G. (2012). Multivariate tail dependence coefficients for Archimedean copulae. In Advanced Statistical Methods for the Analysis of Large Data-Sets, pages 287-296. Springer.

Di Bernardino, E. and Rullière, D. (2013a). Distortions of multivariate distribution functions and associated level curves: Applications in multivariate risk theory. Insurance: Mathematics and Economics, 53(1):190 - 205.

Di Bernardino, E. and Rullière, D. (2013b). On certain transformations of Archimedean copulas: Application to the non-parametric estimation of their generators. Dependence Modeling, 1:1-36.

Di Bernardino, E. and Rullière, D. (2014). Estimation of multivariate critical layers: Applications to hydrological data. Preprint available on HAL Archiv, http://hal.archives-ouvertes.fr/hal-00940089.

Dimitrova, D. S., Kaishev, V. K., and Penev, S. I. (2008). Ged spline estimation of multivariate Archimedean copulas. Computational Statistics 86 Data Analysis, 52(7):3570-3582.

Dobric, J. and Schmid, F. (2005). Nonparametric estimation of the lower tail dependence in bivariate copulas. Journal of Applied Statistics, 32(4):387-407.

Durante, F., Fernández-Sánchez, J., and Pappadà, R. (2014). Copulas, diagonals, and tail dependence. Fuzzy Sets and Systems.

Durante, F., Foschi, R., and Sarkoci, P. (2010). Distorted copulas: Constructions and tail dependence. Communications in Statistics Theory and Methods, 39(12):2288-2301.

Durante, F. and Sempi, C. (2005). Copula and semicopula transforms. International Journal of Mathematics and Mathematical Sciences, 2005(4):645-655.

Durrleman, V., Nikeghbali, A., and Roncalli, T. (2000). A simple transformation of copulas. Technical report, Groupe de Research Operationnelle Credit Lyonnais.

Embrechts, P. and Hofert, M. (2011). Comments on: Inference in multivariate Archimedean copula models. TEST, 20(2):263-270.

Embrechts, P., Klüppelberg, C., and Mikosch, T. (1997). Modelling Extremal Events for Insurance and Finance. Springer, Berlin.

Erdely, A., González-Barrios, J. M., and Hernández-Cedillo, M. M. (2014). Frank's condition for multivariate Archimedean copulas. Fuzzy Sets and Systems, 240(0):131 - 136. Theme: Aggregation Operators.

Frahm, G., Junker, M., and Schmidt, R. (2005). Estimating the tail-dependence coefficient: Properties and pitfalls. Insurance: Mathematics and Economics, 37(1):80 - 100.

Genest, C., Ghoudi, K., and Rivest, L.-P. (1998). Discussion of "understanding relationships using copulas," by edward frees and emiliano valdez, january 1998. North American Actuarial Journal, 2(3):143-149.

Genest, C., Nešlehová, J., and Ziegel, J. (2011). Inference in multivariate Archimedean copula models. TEST, 20(2):223-256.

Goldie, C. and Klüppelberg, C. (1998). Subexponential distributions, pages 435-459. In Adler, R., Feldman, R., Taqqu, M.S. (Eds.), A Practical Guide to Heavy Tails: Statistical Techniques for Analysing Heavy Tailed Distributions. Birkhäuser, Boston.

Hofert, M. (2011). Efficiently sampling nested Archimedean copulas. Computational Statistics $\& 3$ Data Analysis, 55(1):57 - 70.

Hofert, M., Mächler, M., and McNeil, A. (2011). Estimation for Archimedean copulas in high dimensions. Preprint.

Hua, L. and Joe, H. (2011). Tail order and intermediate tail dependence of multivariate copulas. Journal of Multivariate Analysis, 102(10):1454-1471.

Hua, L. and Joe, H. (2012). Tail comonotonicity: Properties, constructions, and asymptotic additivity of risk measures. Insurance: Mathematics and Economics, 51(2):492 - 503.

Joe, H. (1997a). Multivariate models and dependence concepts, volume 73 of Monographs on Statistics and Applied Probability. Chapman \& Hall, London.

Joe, H. (1997b). Multivariate Models and Multivariate Dependence Concepts. Chapman \& Hall/CRC Monographs on Statistics \& Applied Probability. Taylor \& Francis.

Juri, A. and Wüthrich, M. V. (2002). Copula convergence theorems for tail events. Insurance Math. Econom., 30(3):405-420.

Juri, A. and Wüthrich, M. V. (2003). Tail dependence from a distributional point of view. Extremes, 6(3):213-246. 
Klement, E. P., Mesiar, R., and Pap, E. (2005a). Archimax copulas and invariance under transformations. Comptes Rendus Mathematique, 340(10):755 - 758 .

Klement, E. P., Mesiar, R., and Pap, E. (2005b). Transformations of copulas. Kybernetika (Prague), 41(4):425 -434.

Klüppelberg, C. (1988). On subexponential distributions and integrated tails. J. Appl. Probab., 25:132-141.

Klüppelberg, C. (1989a). Estimation of ruin probabilities by means of hazard rates. Insurance: Mathematics and Economics, 8(4):279285.

Klüppelberg, C. (1989b). Subexponential distributions and characterizations of related classes. Prob. Th. Rel. Fields, 82:259-269.

Larsson, M. and Nešlehová, J. (2011). Extremal behavior of Archimedean copulas. Advances in Applied Probability, 43(1):195-216.

Ledford, A. W. and Tawn, J. A. (1997). Modelling dependence within joint tail regions. J. Roy. Statist. Soc. Ser. B, 59(2):475-499.

Li, H. (2009). Orthant tail dependence of multivariate extreme value distributions. Journal of Multivariate Analysis, $100(1): 243$ - 256.

McNeil, A. and Nešlehová, J. (2009). Multivariate Archimedean copulas, d-monotone functions and $l_{1}$-norm symmetric distributions. The Annals of Statistics, 37(5B):3059-3097.

Meyn, S. and Tweedie, R. L. (2009). Markov Chains and Stochastic Stability. Cambridge University Press, New York, NY, USA, 2nd edition.

Michiels, F. and De Schepper, A. (2012). How to improve the fit of Archimedean copulas by means of transforms. Statistical Papers, $53(2): 345-355$.

Morillas, P. M. (2005). A method to obtain new copulas from a given one. Metrika, 61(2):169-184.

Nelsen, R. B. (1999). An introduction to copulas, volume 139 of Lecture Notes in Statistics. Springer-Verlag, New York.

Nelsen, R. B., Quesada-Molina, J. J., Rodríguez-Lallena, J. A., and Úbeda-Flores, M. (2008). On the construction of copulas and quasi-copulas with given diagonal sections. Insurance: Mathematics and Economics, 42(2):473-483.

Schlather, M. (2001). Examples for the coefficient of tail dependence and the domain of attraction of a bivariate extreme value distribution. Statistics \& Probability Letters, 53(3):325 - 329.

Schmidt, R. and Stadtmüller, U. (2006). Non-parametric estimation of tail dependence. Scandinavian Journal of Statistics, 33(2):307335 .

Sibuya, M. (1960). Bivariate extreme statistics. Ann. Inst. Statist. Math., 11:195-210.

Soulier, P. (2009). Some applications of regular variation in probability and statistics. Escuela Venezolana de Matemáticas.

Su, C. and Tang, Q. (2003). Characterizations of heavy-tailed distributions by means of hazard rate. Acta Mat. Appl. Sinica, English Series, 19(1):135-142.

Valdez, E. and Xiao, Y. (2011). On the distortion of a copula and its margins. Scandinavian Actuarial Journal, 4:292-317.

Venter, G. (2001). Tails of copulas. In Proceedings ASTIN Washington, pages 68-113. 